\title{
Upper montane grassland structure within six subranges of Serra do Mar, Southern Brazil ${ }^{1}$
}

\author{
Maurício Bergamini Scheer ${ }^{2,4}$ and Alan Yukio Mocochinski ${ }^{3}$
}

Received: 11.01.2016; accepted: 8.07.2016

\begin{abstract}
Upper montane grassland structure within six subranges of Serra do Mar, Southern Brazil). The phytosociological structure of upper montane grasslands (high altitude grasslands) was studied in six subranges of Serra do Mar. Throughout $324\left(1 \mathrm{~m}^{2}\right)$ plot samples, we identified 195 taxa out of 280 taxa previously found in a floristic survey. Besides the general analysis of these communities, five physiognomies (synusiae) of these grasslands were previously determined based upon the species with greater cover. Cryptangium triquetrum and Croton mullerianus had the highest phytosociological importance value among the upper montane grasslands sampled in the present study. The first species was the most important of the grassy physiognomy of all sampled subranges and the second one of the shrubby physiognomy within three subranges. Chusquea pinifolia, Machaerina austrobrasiliensis, Deschampsia caespitosa, Gleichenella pectitata, Tibouchina dubia, Xyris stenophylla, Eryngium koehnearum and Eriochrysis holcoides were also structurally important. Although considerable species richness has been observed, the dominance of one or few species in the community was common in all subranges and physiognomies. In a brief comparison with upper montane vegetation studies (mainly on rocky outcrops) carried out in Southeastern Brazil, a low sharing of species was verified. Furthermore, the scarcity of studies in the literature regarding floristic and sociological structure of upper montane grasslands hampers a deeper analysis at level of species.
\end{abstract}

Keywords: high altitude grassland, phytosociology, Sea Mountain Range

RESUMO - (Estrutura de campos altomontanos de seis serras no sul do Brasil). A estrutura fitossociológica de Campos Altomontanos foi avaliada em montanhas pertencentes a seis blocos da Serra do Mar, no Estado do Paraná: Serra Gigante, Serra do Ibitiraquire, Serra da Farinha Seca, Serra da Igreja, Serra da Prata e Serra da Pedra Branca do Araraquara. Em 324 parcelas de $1 \mathrm{~m}^{2}$, foram amostrados 195 taxa, dos 280 encontrados em estudo florístico prévio. Além da análise geral dessas comunidades, foram pré-definidas cinco fisionomias vegetacionais (sinúsias) com base nas espécies com provável maior destaque na cobertura. Cryptangium triquetrum e Croton mullerianus apresentaram-se como as principais espécies dos campos altomontanos amostrados na Serra do Mar paranaense. A primeira foi a espécie mais importante em todos os trechos de fisionomia campestre, enquanto a segunda destacou-se na fisionomia subarbustiva em três serras amostradas. Chusquea pinifolia, Machaerina austrobrasiliensis, Deschampsia caespitosa, Gleichenella pectitata, Tibouchina dubia, Xyris stenophylla, Eryngium koehnearum e Eriochrysis holcoides também se destacaram em diversos trechos e fisionomias. Apesar de considerável riqueza, a dominância de uma ou poucas espécies nas comunidades avaliadas foi uma constante em todas as serras e fitofisionomias. Em uma breve comparação com estudos realizados em vegetações altomontanas, principalmente sobre afloramentos de rocha no Sudeste do Brasil, foi verificado um baixo compartilhamento de espécies com os campos altomontanos do presente estudo. A escassez de trabalhos publicados sobre a florística e a estrutura fitossociológica desses campos, dificulta análises mais aprofundadas em nível de espécie.

Palavras-chave: Campos de altitude, Campos de "alta"altitude, fitossociologia, Serra do Mar

\section{Introduction}

Standing out in a context of montane and upper montane forests, the natural landscape at the top of several mountain ranges is largely occupied by grassland vegetation. The highland regions of both Serra do Mar and Serra da Mantiqueira present about $350 \mathrm{~km}^{2}$ of high altitude grasslands (Safford 1999a).

1. Parte deste artigo é parte atualizada da Dissertação de Mestrado do segundo Autor

2. SANEPAR - Companhia de Saneamento do Paraná (APD/CETS/DMA), Rua Engenheiro Antônio Batista Ribas, 151, Tarumã, 82800130 Curitiba, PR, Brasil

3. ICMBio - Instituto Chico Mendes de Conservação da Biodiversidade ICMBio, Área de Proteção Ambiental de Guaraqueçaba, Praça Carlos Cavalcanti, 48, Estação Ferroviária, 83370-000 Antonina, PR, Brasil

4. Corresponding author: mauriciobs@sanepar.com.br 
Many descriptions for the various grassland formations of plateau surfaces and mountain ranges of Brazil have been reported in the literature by several authors. However, these descriptions often result in confusion, redundant and inaccurate categorization of such environments, especially for those higher level mountain ranges (e.g. alpine, mountaintop and steppelike grasslands, upper montane or high elevation refuges, ecological refuges, upper montane herbaceous plant refuges, rocky grasslands (rocky outocrop vegetation), high-altitude rupestrian grasslands, the Atlantic Forest biome grasslands, altitude grasslands (campos de altitude, a generic term), upper montane grasslands (campos de "alta" altitude or "campos altomontanos”, etc.) (e.g. Rizzini 1979, Ferri 1980, Veloso et al. 1991, IBGE 1992, Martinelli 1996, Safford 1999a, b, Tramujas 2000, Roderjan et al. 2002, Caiafa \& Silva 2005, 2007, Garcia \& Pirani, 2005, Ribeiro et al. 2007, Mocochinski \& Scheer 2008, IBGE 2012, Vasconcelos 2011, Scheer et al. 2011a, 2013a, b, 2014, Meireles et al. 2014, Alves et al. 2014, Tinti et al. 2015).

The Brazilian CONAMA Resolution 10/1993 had already ascribed "campos de altitude" to the montane and upper montane levels occurring in litholic ridges from high-altitude mountains. CONAMA Resolution 423/2010, in order to link Federal Law no. $11.428 / 2006$, regarded the term "campos de altitude" not only to upper montane environments, but also to the montane Atlantic Forest biome.

Quite often, the term "campos rupestres" (rocky outcrop vegetation or rupestrian grasslands) is used by some authors to the vegetation which predominantly occurs on very shallow soils on quartzite and sandstone rocks (among others), usually at montane and upper montane levels (Ferri 1980, Vasconcelos 2011, among others). Despite having similar physiognomy and sharing some taxa, the rock fields and "high-altitude graslands" should be treated as distinct based upon differences in geological substrates, plant species composition, climatic and phytogeographic domains (Vasconcelos 2011, Alves et al. 2014). However, regarding the term "montane and upper montane rupicolous/rupestrian vegetation" could also be attributed to the transitional sites with incipient substrates and/or the primary succession colonizing rock outcrops close to the upper montane grasslands of Serra do Mar (Coastal Mountain Range). As a matter of fact, both of them can be considered as upper montane vegetation refuges (Veloso et al. 1991), generally occurring in thin histic topsoil substrates over granitic and gneissic/migmatitic rocks, and therefore not necessarily over quartzite or sandstone rocks by leaving the physiognomy of such upper montane grasslands or even the rock grasslands uncovered. Furthermore, the term "altitude", although widely used (sometimes even by law) to determine montane and upper montane levels, is quite inaccurate since every location has a specific altitude which needs to be specified so that confusion and generalizations are avoided. Also, the terms "rupestrian" or "rupicolous" denote the neighboring or interspersed rock outcrops over topsoils or incipient substrates, which may occur on several lithologies, and for this reason the vegetation type should be added. The term "fields", adopted by IBGE (Brazilian Institute of Geography and Statistics), is more comprehensive: herbaceous and shrubby vegetation, consisting primarily of graminoids and scattered bushes. Therefore, the above terminology requires better specification. We aimed at differentiating the grasslands (CONAMA Resolution 423/2010) occurring in montane levels (commonly found in hinterland uplands) from the "high" altitude grasslands, which are predominantly Haplic and Folic Histosols that also feature "rupestrian areas" with transitional rock outcrops. To this end, we adopted in the present study the term "Upper Montane Grasslands", abiding by the parameters set for forests according to IBGE Vegetation Classification System. They can also be considered as upper montane vegetation refuges based on the concept that they dominated larger areas in the last glacial periods. Currently, along with the transition from rupestrian flora to rocky outcrops, they are different from the dominant context of forests. On the other hand, we have to point out that these ecosystems are current patterns of where they are located, arising from morphogenesis and pedogenesis processes which resulted from climate change and geomorphology up to date.

Scheer et al. $(2011,2013)$ explain the occurrence of upper montane grasslands in comparison to upper montane forests, based on the soil factor through the greater thicknesses of histic horizons in comparison to mineral ones since the characteristics of both soils are strongly related to the climate and geomorphology. High altitudes bring about lower temperatures, which favors a slower decomposition of litter, forming then such organic horizons. These conditions combined with the strong winds seem to promote herbaceous and shrubby species instead of tree species because these horizons have no structure to provide stability for the roots to sustain large trees. Moreover, grazing 
by cattle and wildfires caused by human activity damage the physiognomic characteristics of some upper montane grasslands, even though many authors considered these environments as anthropic fields such as "Pampas" in Southern Brazil (Overbeck et al. 2007, Behling et al. 2009) and Campos Gerais (Safford 2001) as well as high-altitude grasslands in Southeastern Brazil. Some upper montane grasslands in the Southern and Southeastern Brazil have endured wildfires resulted from illegal agro-pasture activities of neighboring properties. As a matter of fact, only $0.5 \%$ of wildfires in the Southeastern mountains appear to be natural (Aximoff 2001), which has severely altered their floristic and pedological composition. In many upper montane grasslands of Serra do Mar, the occurrence of some specialized underground organs with the function to support grazing and fires can be only an acquired inheritance during drier past climates. It does not mean that these current mature (old growth) grasslands need fire for its maintenance.

Many areas of montane and upper montane forests have also been devastated by man-made fires, losing their ecological resilience and beginning a colonization process along with countryside-like physiognomy, which causes a disruption and distortion regarding natural grasslands.

The building processes for the formation of upper montane grasslands are complex and date back to an ancient landscape by the end of the Pleistoceneepoch, when dry-cold severe weather dominated the Southeastern region of the South American continent (Safford 1999b). According to Safford (1999a), the high number of endemic species in this formation indicates that these environments are relatively ancient and not only the result of recent anthropogenic disturbances. There is Palaeobotanical evidence that suggests that the mountain ridges of Southeastern Brazil have been uninterruptedly covered by upper montane grasslands since the end of the Pleistocene (Behling 1997a, b). A similar situation must have occurred to the regions immediately to the South, although back then some exposed mountain ridges may have been largely bare or covered only by precursor rupestrian vegetation, being colonized (or recolonized) throughout the Holocene (Scheer et al. 2012, 2014).

Unlike the countryside-like montane grasslands (such as "Pampas"), the difficulty of access and the adversity of environmental conditions may have safeguarded upper montane grasslands from anthropogenic degradation processes. Thus, they are rare well-preserved areas of nature in the South and
Southeast of Brazil. However, their preservation has been constantly threatened by anthropogenic processes such as wildfires, biological contamination as well as disorderly tourism development (Mocochinski \& Scheer 2008). While considering the high levels of endemism, the uniqueness of their ecological processes (high carbon stocks in the soil per unit area, soil retention and regulation of water flows, slope protection, etc., Scheer et al. 2011, 2013) as well as their restricted occurrence within a few tens of square kilometers in the State of Paraná, it is clear the importance of action plans for the conservation of such vegetation. Hence, improved basic knowledge is crucial, such as structural and floristic composition which are still scarce. By acknowledging this gap in knowledge about the biological diversity in the Southern region of Brazil, besides complementing and updating the floristic study reported by Mocochinski \& Scheer (2008), we aimed at presenting the phytosociological structure of upper montane grasslands from several mountains belonging to six subranges of Serra do Mar in Paraná and their similarity. Additionally, we conducted a brief search for studies which might include similar sites in other regions of Brazil. Gathering information and knowledge about the most dominant and frequent species in these formations as well as their different vegetation types in Paraná play a key role for further research on such important environments.

\section{Material and methods}

Study areas - The six sampled subranges belong to Serra do Mar in the State of Paraná, Southern Brazil. The geographic coordinates are between latitudes $25^{\circ} 56^{\prime} \mathrm{S}$ and $25^{\circ} 08^{\prime} \mathrm{S}$ and longitudes $48^{\circ} 52^{\prime} \mathrm{W}$ and $48^{\circ} 30^{\prime} \mathrm{W}$. The subranges are regionally known as: Serra do Ibitiraquire, Serra da Igreja, Serra da Prata, Serra Gigante, Serra da Farinha Seca (Graciosa) and Serra da Pedra Branca do Araraquara (figure 1). Such study areas were chosen based on the representativeness of this formation occurrence in Paraná and a number of outstanding differences: for instance, altitude (between 950 and $1870 \mathrm{~m}$ asl), extension and altitudinal ranges of the formation, geographical position, continentality, etc. (table 1).

The climate is classified as Cfb by the KöppenGeiger system: humid subtropical climate, average temperature of the coldest month below $18{ }^{\circ} \mathrm{C}$ and above $-3{ }^{\circ} \mathrm{C}$, average temperature of the warmest month below $22{ }^{\circ} \mathrm{C}$. Roderjan \& Grodski (1998) observed absolute minimum temperature of $-5{ }^{\circ} \mathrm{C}$, 
annual average of $13.4{ }^{\circ} \mathrm{C}$ and absolute maximum of $30^{\circ} \mathrm{C}$ to upper montane levels, in forest environment, in Morro Anhangava in the municipality of Quatro Barras, Paraná. The rate of precipitation in Serra do Mar is well distributed throughout the year and show wide variation depending on the topography. Measurements in the coastal region surpass 2,000 mm annual precipitation and on the slopes of the subranges the values reach $3,500 \mathrm{~mm}$. As for hinterland uplands, surpassing mountain ranges, annual precipitation may reach 1,500 mm (Maack 1968).

As previously mentioned, the current occurrence of upper montane grasslands over the Upper Montane Atlantic Dense Rain (Cloud) Forest is regulated by the substrate thickness relationships between (histic) organic and mineral horizons, which resulted from both climate and geomorphology. The upper montane grasslands are in predominantly Haplic and Folic Histosols as well as Histic Litholic Entisols, which either have no mineral horizons or their occurrence typically starts at a depth below $40 \mathrm{~cm}$ or even in places where histic horizons have a lithic contact within the depth between 20 and $40 \mathrm{~cm}$. The sites where histic horizons have less thickening there are better conditions for the upper montane forest to be established (Scheer et al. 2013a, b), then taking place in Litholic Entisols (typically dystrophic and typically humic) (Roderjan 1994, Rocha 1999, Vaschenko et al. 2007) and Gleysols (Wisniewski et al. 2005, Scheer et al. 2013a), Regosols (Scheer et al. 2013b) as well as Cambisols (Roderjan 1994, Rocha 1999). These extremely acid soils have high organic matter content, low base saturation and high exchangeable aluminum content. In lower altitude or even isolated mountains in southern Brazil (e.g. Serra Gigante), the typical upper montane grasslands may occur more significantly close to the ridges, just over $1000 \mathrm{~m}$ asl, which does not occur in higher mountains (e.g. above $1300 \mathrm{~m}$ asl). As a matter of fact, such occurrences at lower altitudes are due to geomorphological and soil characteristics, as well as mass-elevation effect (e.g. lower temperatures in comparison with higher and more extensive subranges) also known as the Massenerhebung effect (Grubb 1971, Flenley 1995, Han et al. 2012).

As for higher subranges such as Serra do Ibitiraquire, the upper montane grasslands have a more extensive range, being proportionally higher (above 1,400 $\mathrm{m}$ asl), is interweaving with the occurrence of upper montane forests and rocky outcrops, by evidencing the important role of other environmental factors (table 1).

Phytosociological study - In order to study the sociological structure of the plant communities, we sampled 324 plots $(1 \times 1 \mathrm{~m})$ in the six subranges. A different number of sample plots were set up on each subrange based upon the field area length, the number of ridges per subrange, accessibility on each subrange as well as the phytophysiognomy in the field.

Aiming to refine the approach to the structure of the communities, the samples were classified into categories, hereby treated as vegetation types or physiognomy ("synusiae"), found well preserved and in an advanced, (mature or old growth) succesion stage. This typological pre-stratification (Péllico Neto $\&$ Brena 1997) was used to obtain particular data for

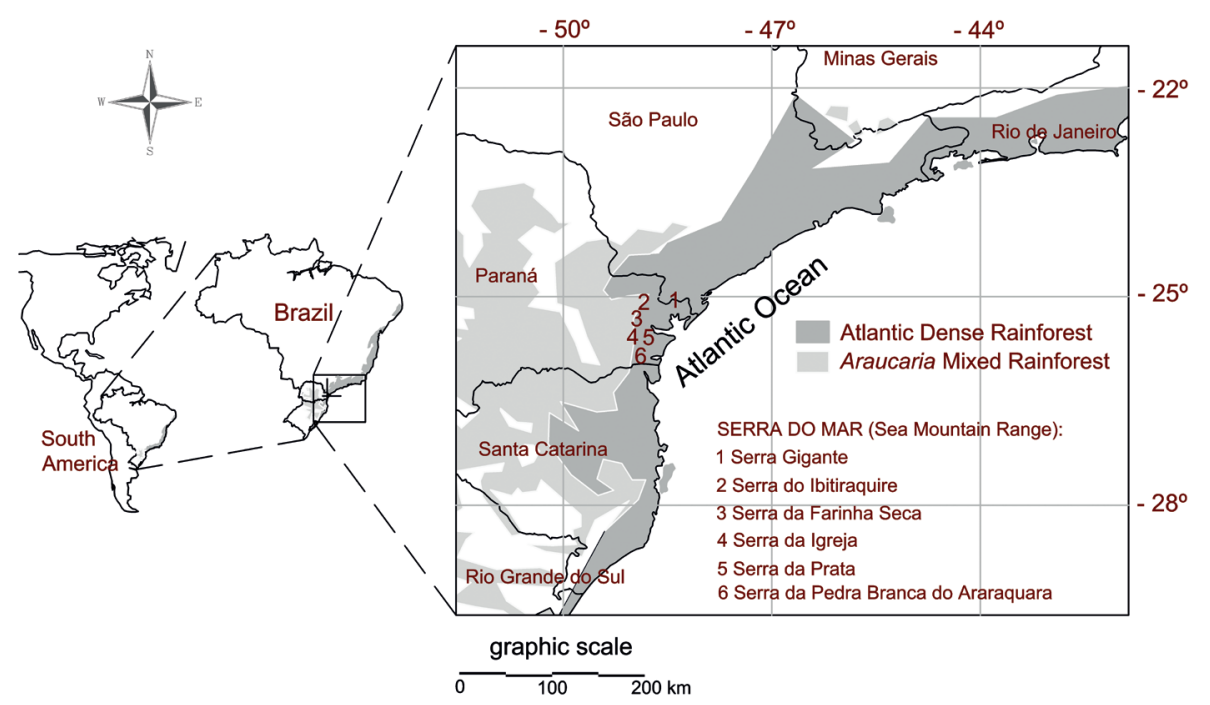

Figure 1. Location of the Subranges where the upper montane grasslands were sampled. 
each vegetation physiognomy, easily characterized and recognized in the field, which enabled more accurate estimates of phytosociological descriptors. The characterization was based upon the most common species with greater cover or similar habitats, thereby visually defining the synusiae.

Five physiognomies were determined: 1 . Shrubby, when the species with greater cover were visibly shrubs; 2. Grassy, when the species with greater cover were visibly graminoids; 3. Chusquea mimosa; 4. Chusquea pinifolia; 5. Machaerina austrobrasiliensis; when these species were visibly and clearly the most dominant ones.

All plant taxa found in the plots were registered in field records. We also recorded the maximum height and the predominant height of each species per sample plot as well as the respective percentage of the species cover (adaptation of the method reported by Braun-Blanquet 1979), based on the estimated vertical projection of the aerial parts of the plants sampled on the ground. To determine the predominant height, we took into consideration the most photosynthetically active portion of the species found within the plots sampled. To aid measurement of species cover, the plots were assembled with metric graduation measuring tapes. No attempt was made to record species density due to the difficulty to determine the number of different specimens found in the field, especially graminoids. Plant material samples were collected from each plot in order to compose the floristic list (published by Mocochinski \& Scheer 2008) and also to identify the ones yet unknown by the authors. However, for some of those as yet unknown we used morpho-species

Table 1. Features of the sampled subranges of Serra do Mar (Sea Mountain Range) in Southern Brazil

\begin{tabular}{|c|c|c|c|c|c|}
\hline Subrange & $\begin{array}{l}\text { Highest ridge } \\
\text { point (m asl) }\end{array}$ & $\begin{array}{c}\text { Location } \\
\text { (Municipalities) }\end{array}$ & $\begin{array}{c}\text { Lithology } \\
\text { (PRÓ-ATLÂNTICA, } \\
\text { 2002) }\end{array}$ & Features & Protected Area Unit \\
\hline
\end{tabular}

\begin{tabular}{|c|c|c|c|c|}
\hline Gigante & 1069 & $\begin{array}{l}\text { Guaraqueçaba } \\
\text { (PR) e Cananéia } \\
\text { (SP) }\end{array}$ & $\begin{array}{c}\text { Gneissic-migmatitic } \\
\text { rocks } \\
\text { (foliated granitic) }\end{array}$ & $\begin{array}{l}\text { Upper montane grasslands restricted } \\
\text { to the vicinity of ridges, occurring } \\
\text { more significantly over } 1,050 \mathrm{~m} \text { APA de } \\
\text { asl. Coastal plain isolated subrange. Guaraqueçaba e PE } \\
\text { No contact point with the plateau } \\
\text { (hinterland upland) }\end{array}$ \\
\hline do Ibitiraquire & 1877 & $\begin{array}{c}\text { Campina } \\
\text { Grande do Sul e } \\
\text { Antonina (PR) }\end{array}$ & $\begin{array}{c}\text { Alkali granites } \\
\text { (Granite-Graciosa) }\end{array}$ & $\begin{array}{l}\text { Approximately } 790 \text { hectares of upper } \\
\text { montane grassland cover (Roderjan, PE do Pico Paraná } \\
\text { 1999). Occurrence over } 1,350 \mathrm{~m} \text { asl. e Roberto Ribas } \\
\text { There are } 13 \text { mountains with altitude } \\
\text { greater than } 1,500 \mathrm{~m} \text { asl }\end{array}$ \\
\hline $\begin{array}{l}\text { da Farinha } \\
\text { Seca }\end{array}$ & 1457 & $\begin{array}{l}\text { Quatro Barras e } \\
\text { Morretes (PR) }\end{array}$ & $\begin{array}{c}\text { Alkali granites } \\
\text { (Granite-Graciosa) }\end{array}$ & $\begin{array}{l}\text { Upper montane grasslands } \\
\text { occurring over } 1,300 \mathrm{~m} \text { asl. It has } \mathrm{PE} \text { da Graciosa } \\
\text { flat-topped ridges. }\end{array}$ \\
\hline da Igreja & 1376 & Morretes (PR) & $\begin{array}{l}\text { Alcali granites } \\
\text { (Alkali-granite) }\end{array}$ & $\begin{array}{l}\text { Upper montane grasslands } \\
\text { occurring over } 1,250 \mathrm{~m} \text { asl. It has } \text { PARNA Guaricana } \\
\text { flat-topped elevations }\end{array}$ \\
\hline da Prata & 1502 & $\begin{array}{c}\text { Morretes, } \\
\text { Paranaguá e } \\
\text { Guaratuba (PR) }\end{array}$ & $\begin{array}{l}\text { Gneissic-migmatitic } \\
\text { rocks } \\
\text { (foliated granitic) }\end{array}$ & $\begin{array}{l}\text { Upper montane grasslands } \\
\text { occurring over } 1,300 \mathrm{~m} \text { asl. Coastal } \\
\text { plain isolated-subrange, no contact } \\
\text { point with the plateau }\end{array}$ \\
\hline $\begin{array}{r}\text { da Pedra Branca } \\
\text { do Araraquara }\end{array}$ & 1222 & Guaratuba (PR) & $\begin{array}{l}\text { Granite-gneissic } \\
\text { rocks }\end{array}$ & $\begin{array}{l}\text { Upper montane grasslands occurring APA de Guaratuba } \\
\text { over } 1,150 \mathrm{~m} \text { asl }\end{array}$ \\
\hline
\end{tabular}

APA - Environmental Protection Area

PE - State Park

PARNA - National Park

1 - PRÓ-ATLÂNTICA 2002

2 - Roderjan 1999,

Source: Mocochinski \& Scheer (2008) 
identification, since the specific determination was not possible due to the lack of suitable source material for this purpose. The reference material was deposited in the collection of Museu Botânico Municipal in Curitiba (MBM) and duplicates were donated to the Herbarium of Universidade Federal do Paraná (UFPR-UPCB) as well as to Escola de Florestas de Curitiba (EFC). Taxa of our phytosociological survey were reviewed according to the List of Species of the Brazilian Flora 2020 (Flora of Brazil under construction 2016) as well as the Brazilian Catalogue of Plants and Fungi (Forzza et al. 2010).

The final graphical presentation relating to the phytosociological importance of the species in each physiognomy within the six sampled subranges was developed by AMADO 1:01 CISIA-program. The cluster analysis was performed in STATIGRAPHICS PLUS-program.

\section{Results and Discussion}

Structure of upper montane grasslands in Serra do Ibitiraquire

Serra do Ibitiraquire - Physiognomy: shrubby - We found 94 species within the 61 sampled plots and the community had an absolute plant cover of $124.7 \mathrm{~m}^{2} .100^{-}$ ${ }^{1} \mathrm{~m}^{2}$ (considered here the sum of the cover of all sampled species), especially Croton mullerianus with the highest importance percentage (table 2). Such emphasis was mainly due to the cover percentage (36.5\%), more than double if compared to the second species, Cryptangium triquetrum. The main six species accounted for almost half the percentage of the community. Furthermore, C. mullerianus and C. triquetrum alone accounted for more than half the community relative cover.

Chusquea pinifolia stood out in the upper strata of the community with predominant height (average of $87.8 \mathrm{~cm}$ ) and relative cover of 5.9\%. Chusquea mimosa also stood out in the upper strata with prevailing height (average of $85 \mathrm{~cm}$ ), however, it was found in only $20 \%$ of the sampled plots.

The species with predominant height lower than $30 \mathrm{~cm}$ accounted for less than $5 \%$ the community relative cover. Whereas, the species with predominant height between 30 and $60 \mathrm{~cm}$ accounted for $84 \%$ the relative cover, of which four out of the five most important species of such community were found in this stratum. The species with prevailing height above $60 \mathrm{~cm}$ accounted for $11 \%$ the relative cover, especially C. pinifolia, C. mimosa and Machaerina austrobrasiliensis.
Serra do Ibitiraquire - Physiognomy: grassy - 99 species (mainly graminoids) were found within the 64 sampled plots (table 3 ). The community had an absolute plant cover of $109 \mathrm{~m}^{2} .100^{-1} \mathrm{~m}^{2}$. C. triquetrum showed the highest percentage $(16,4 \%)$ and relative cover of $26.5 \%$, which represents over a quarter of the community. The difference for the other species was not as great as in the shrubby physiognomy, however, we observed that only seven species accounted for more than $50 \%$ the highest importance percentage of the community. The three main species accounted for almost $50 \%$ the community relative cover.

Cryptangium triquetrum, dominating nearly a third of the community showed a predominant height of $54.6 \mathrm{~cm}$, which is higher than the other nine most important species. Thus, it characterized the physiognomy of the vegetation by creating a typical "graminoid" carpet (although Cyperaceae).

On this physiognomy, the species' prevailing height between 0 and $30 \mathrm{~cm}$ comprised $5 \%$ of the relative cover. The species with average hight between 30 and $60 \mathrm{~cm}$ comprised $47 \%$ of the relative cover. As for the species with average height greater than $60 \mathrm{~cm}$ comprised $48 \%$ of the relative cover.

Serra do Ibitiraquire - Physiognomy: Chusquea pinifolia - We found 85 species and an absolute cover of $125 \mathrm{~m}^{2} \cdot 100^{-1} \mathrm{~m}^{2}$ within the 56 sampled plots with dominance of Chusquea pinifolia (caratuva). This species comprised more than a quarter of the importance percentage and almost half of the community relative cover (table 4). Once again $C$. mullerianus and Cryptangium triquetrum were among the four most important species in the sampled plot. Those, along with Machaerina austrobrasiliensis and Alstroemeria amabilis accounted for more than half the importance percentage.

This physiognomy has higher size due to the dominance of $C$. pinifolia, which showed height average of $78 \mathrm{~cm}$. The gradual increase in height of such physiognomy is likely a result of the relative thickness of subsurface mineral horizons, below histosol horizons (histic) (Scheer et al. 2014). Another strata was observed some $50 \mathrm{~cm}$ afar, in which Croton mullerianus, Rhynchospora exaltata and C. triquetrum were dominant. We also observed the occurrence of important "graminoid" species such as C. triquetrum, Deschampsia caespitosa, Xyris stenophylla and Eriochrysis holcoides within the community.

Serra do Ibitiraquire - Physiognomy: Chusquea mimosa - 46 species were found in the nine sampled 
Table 2. Phytosociological parameters of the shrubby physiognomy of the upper montane grasslands in Serra do Ibitiraquire (Mountain Range), $\mathrm{n}=61$. FA: absolute frequency (\%); Co: absolute cover (\%); FR: relative frequency (\%); CoR: relative cover (\%); PI: importance percentage (\%); Hpre: predominant height (cm); Hmax: maximum height $(\mathrm{cm})$.

\begin{tabular}{|c|c|c|c|c|c|c|c|c|}
\hline & Species & FA & Co & FR & CoR & PI & Hpre & Hmax \\
\hline 1 & Croton mullerianus & 93.4 & 45.46 & 9.39 & 36.47 & 22.93 & 45.5 & 54.5 \\
\hline 2 & Cryptangium triquetrum & 82.0 & 19.61 & 8.24 & 15.73 & 11.98 & 58.0 & 74.8 \\
\hline 3 & Chusquea pinifolia & 36.1 & 7.36 & 3.62 & 5.90 & 4.76 & 87.8 & 116.2 \\
\hline 4 & Mimosa congestifolia & 34.4 & 5.25 & 3.46 & 4.21 & 3.83 & 54.0 & 59.8 \\
\hline 5 & Hesperozygis nitida & 31.1 & 4.05 & 3.13 & 3.25 & 3.19 & 36.7 & 45.7 \\
\hline 6 & Machaerina austrobrasiliensis & 31.1 & 2.64 & 3.13 & 2.12 & 2.62 & 61.4 & 77.1 \\
\hline 7 & Rhynchospora exaltata & 27.9 & 3.05 & 2.80 & 2.45 & 2.62 & 52.5 & 66.1 \\
\hline 8 & Chusquea mimosa & 19.7 & 2.95 & 1.98 & 2.37 & 2.17 & 85.1 & 114.5 \\
\hline 9 & Deschampsia caespitosa & 21.3 & 2.25 & 2.14 & 1.80 & 1.97 & 54.5 & 60.6 \\
\hline 10 & Utricularia reniformis & 26.2 & 1.48 & 2.64 & 1.18 & 1.91 & 19.1 & 21.7 \\
\hline 11 & Eryngium koehneanum & 19.7 & 2.13 & 1.98 & 1.71 & 1.84 & 45.1 & 56.3 \\
\hline 12 & Gaylussacia brasiliensis var brasiliensis & 26.2 & 1.25 & 2.64 & 1.00 & 1.82 & 41.0 & 47.2 \\
\hline 13 & Vernonanthura montevidensis & 27.9 & 1.02 & 2.80 & 0.82 & 1.81 & 49.2 & 54.2 \\
\hline 14 & Symphyopappus cuneatus & 23.0 & 1.59 & 2.31 & 1.28 & 1.79 & 50.7 & 57.4 \\
\hline 15 & Xyris stenophylla & 21.3 & 1.25 & 2.14 & 1.00 & 1.57 & 36.3 & 44.8 \\
\hline 16 & Mimosa prionopus & 13.1 & 1.95 & 1.32 & 1.56 & 1.44 & 36.6 & 45.6 \\
\hline 17 & Baccharis crispa & 21.3 & 0.87 & 2.14 & 0.70 & 1.42 & 48.0 & 54.0 \\
\hline 18 & Poaceae 5 & 21.3 & 0.72 & 2.14 & 0.58 & 1.36 & 14.6 & 17.6 \\
\hline 19 & Sticherus sp. & 11.5 & 1.67 & 1.15 & 1.34 & 1.25 & 50.0 & 54.3 \\
\hline 20 & Leandra sulfurea & 19.7 & 0.57 & 1.98 & 0.46 & 1.22 & 37.6 & 41.5 \\
\hline 21 & Myrsine altomontana & 16.4 & 0.97 & 1.65 & 0.78 & 1.21 & 59.9 & 62.8 \\
\hline 22 & Siphoneugena reitzii & 14.8 & 1.02 & 1.48 & 0.82 & 1.15 & 57.7 & 61.3 \\
\hline 23 & Poaceae 15 & 16.4 & 0.69 & 1.65 & 0.55 & 1.10 & 47.1 & 56.0 \\
\hline 24 & Leandra sp. & 18.0 & 0.26 & 1.81 & 0.21 & 1.01 & 34.4 & 36.9 \\
\hline 25 & Eriochrysis holcoides & 13.1 & 0.80 & 1.32 & 0.64 & 0.98 & 34.0 & 43.3 \\
\hline 26 & Lycopodium clavatum & 11.5 & 0.95 & 1.15 & 0.76 & 0.96 & 28.0 & 35.4 \\
\hline 27 & Gaultheria serrata var. organensis & 14.8 & 0.48 & 1.48 & 0.38 & 0.93 & 41.9 & 45.0 \\
\hline 28 & Galianthe gertii & 13.1 & 0.66 & 1.32 & 0.53 & 0.92 & 36.5 & 39.3 \\
\hline 29 & Alstroemeria amabilis & 9.8 & 0.69 & 0.99 & 0.55 & 0.77 & 51.5 & 57.7 \\
\hline 30 & Blechnum cordatum & 9.8 & 0.54 & 0.99 & 0.43 & 0.71 & 43.5 & 47.2 \\
\hline 31 & Dyckia reitzii & 9.8 & 0.52 & 0.99 & 0.42 & 0.70 & 25.8 & 29.8 \\
\hline 32 & Mimosa eurystegia & 8.2 & 0.72 & 0.82 & 0.58 & 0.70 & 36.0 & 42.0 \\
\hline 33 & Rhynchospora sp. 1 & 6.6 & 0.84 & 0.66 & 0.67 & 0.66 & 44.8 & 50.3 \\
\hline 34 & Eriocaulon ligulatum & 9.8 & 0.41 & 0.99 & 0.33 & 0.66 & 20.3 & 24.8 \\
\hline 35 & Tibouchina hospital & 9.8 & 0.30 & 0.99 & 0.24 & 0.61 & 45.0 & 47.3 \\
\hline 36 & Galium hypocarpium ssp. Indecorum & 9.8 & 0.28 & 0.99 & 0.22 & 0.61 & 29.7 & 31.7 \\
\hline 37 & Lycopodium sp. & 8.2 & 0.46 & 0.82 & 0.37 & 0.60 & 27.6 & 35.2 \\
\hline 38 & Myrcia hartwegiana & 8.2 & 0.39 & 0.82 & 0.32 & 0.57 & 54.4 & 60.8 \\
\hline 39 & Tibouchina dúbia & 8.2 & 0.39 & 0.82 & 0.32 & 0.57 & 34.0 & 38.0 \\
\hline 40 & Cyperaceae 1 & 9.8 & 0.18 & 0.99 & 0.14 & 0.57 & 45.8 & 53.7 \\
\hline
\end{tabular}


Table 2 (continuation)

\begin{tabular}{|c|c|c|c|c|c|c|c|c|}
\hline & Species & FA & Co & FR & $\mathrm{CoR}$ & PI & Hpre & Hmax \\
\hline 41 & Achyrocline satureioides & 8.2 & 0.23 & 0.82 & 0.18 & 0.50 & 36.2 & 43.2 \\
\hline 42 & Poaceae 14 & 8.2 & 0.18 & 0.82 & 0.14 & 0.48 & 39.8 & 40.8 \\
\hline 43 & Dichanthelium sabulorum & 8.2 & 0.15 & 0.82 & 0.12 & 0.47 & 50.2 & 43.2 \\
\hline 44 & Phalocallis geniculata & 8.2 & 0.13 & 0.82 & 0.11 & 0.46 & 62.4 & 68.8 \\
\hline 45 & Baccharis angusticeps & 4.9 & 0.46 & 0.49 & 0.37 & 0.43 & 46.0 & 51.3 \\
\hline 46 & Sticherus pruinosus & 4.9 & 0.33 & 0.49 & 0.26 & 0.38 & 48.7 & 49.3 \\
\hline 47 & Lycopodium thyoides & 4.9 & 0.30 & 0.49 & 0.24 & 0.37 & 32.0 & 36.3 \\
\hline 48 & Gaylussacia arassatubensis & 4.9 & 0.23 & 0.49 & 0.18 & 0.34 & 34.3 & 35.7 \\
\hline 49 & Axonopus sp. & 4.9 & 0.16 & 0.49 & 0.13 & 0.31 & 15.7 & 21.3 \\
\hline 50 & Ericaceae 1 & 4.9 & 0.16 & 0.49 & 0.13 & 0.31 & 28.7 & 38.0 \\
\hline 51 & Gaultheria serrata & 4.9 & 0.15 & 0.49 & 0.12 & 0.31 & 37.7 & 48.7 \\
\hline 52 & Miconia sp. & 4.9 & 0.15 & 0.49 & 0.12 & 0.31 & 29.3 & 31.3 \\
\hline 53 & Doryopteris crenulans & 4.9 & 0.10 & 0.49 & 0.08 & 0.29 & 22.7 & 23.3 \\
\hline 54 & Trixis lessingii & 4.9 & 0.10 & 0.49 & 0.08 & 0.29 & 15.3 & 16.0 \\
\hline 55 & Mimosa tucumensis & 3.3 & 0.25 & 0.33 & 0.20 & 0.26 & 40.5 & 49.0 \\
\hline 56 & Ilex microdonta & 1.6 & 0.43 & 0.16 & 0.34 & 0.25 & 100.0 & 110.0 \\
\hline 57 & Poaceae 16 & 3.3 & 0.15 & 0.33 & 0.12 & 0.22 & 58.5 & 62.0 \\
\hline 59 & Chascolytrum calotheca & 3.3 & 0.13 & 0.33 & 0.11 & 0.22 & 70.5 & 78.0 \\
\hline 58 & Ericaceae 2 & 3.3 & 0.13 & 0.33 & 0.11 & 0.22 & 48.0 & 49.5 \\
\hline 60 & Sisyrinchium vaginatum & 3.3 & 0.10 & 0.33 & 0.08 & 0.20 & 45.0 & 45.0 \\
\hline 61 & Melastomataceae 1 & 3.3 & 0.07 & 0.33 & 0.05 & 0.19 & 32.5 & 35.0 \\
\hline 62 & Sisyrinchium sp. & 3.3 & 0.05 & 0.33 & 0.04 & 0.18 & 41.0 & 45.5 \\
\hline 63 & Poaceae 13 & 3.3 & 0.03 & 0.33 & 0.03 & 0.18 & 24.0 & 24.0 \\
\hline 64 & Escallonia laevis & 1.6 & 0.23 & 0.16 & 0.18 & 0.17 & 65.0 & 67.0 \\
\hline 65 & Danthonia secundiflora & 1.6 & 0.16 & 0.16 & 0.13 & 0.15 & 59.0 & 92.0 \\
\hline 66 & Rhynchospora sp. 2 & 1.6 & 0.16 & 0.16 & 0.13 & 0.15 & 15.0 & 24.0 \\
\hline 67 & Quesnelia imbricata & 1.6 & 0.13 & 0.16 & 0.11 & 0.13 & 35.0 & 37.0 \\
\hline 68 & Vriesea hoehneana & 1.6 & 0.10 & 0.16 & 0.08 & 0.12 & 36.0 & 38.0 \\
\hline 69 & Zygopetalum maculatum & 1.6 & 0.10 & 0.16 & 0.08 & 0.12 & 22.0 & 22.0 \\
\hline 70 & Symphyopappus sp. 1 & 1.6 & 0.08 & 0.16 & 0.07 & 0.12 & 67.0 & 75.0 \\
\hline 71 & Lycopodium sp. 2 & 1.6 & 0.07 & 0.16 & 0.05 & 0.11 & 22.0 & 47.0 \\
\hline 72 & Myrciaria tenella & 1.6 & 0.07 & 0.16 & 0.05 & 0.11 & 83.0 & 83.0 \\
\hline 73 & Stevia claussenii & 1.6 & 0.07 & 0.16 & 0.05 & 0.11 & 15.0 & 20.0 \\
\hline 74 & Gesneriaceae indet. & 1.6 & 0.05 & 0.16 & 0.04 & 0.10 & 47.0 & 47.0 \\
\hline 75 & Poaceae 10 & 1.6 & 0.05 & 0.16 & 0.04 & 0.10 & 24.0 & 34.0 \\
\hline 76 & Poaceae 11 & 1.6 & 0.05 & 0.16 & 0.04 & 0.10 & 66.0 & 73.0 \\
\hline 77 & Smilax campestris & 1.6 & 0.05 & 0.16 & 0.04 & 0.10 & 43.0 & 43.0 \\
\hline 78 & Symphyopappus sp. 2 & 1.6 & 0.05 & 0.16 & 0.04 & 0.10 & 60.0 & 60.0 \\
\hline 79 & Symplocos corymboclados & 1.6 & 0.05 & 0.16 & 0.04 & 0.10 & 42.0 & 42.0 \\
\hline 80 & Coccocypselum condalia & 1.6 & 0.03 & 0.16 & 0.03 & 0.10 & 22.0 & 27.0 \\
\hline 81 & Esterhazya splendida & 1.6 & 0.03 & 0.16 & 0.03 & 0.10 & 50.0 & 61.0 \\
\hline 82 & Gaultheria sp. 1 & 1.6 & 0.03 & 0.16 & 0.03 & 0.10 & 68.0 & 68.0 \\
\hline
\end{tabular}


Table 2 (continuation)

\begin{tabular}{llccccccc}
\hline & Species & FA & Co & FR & CoR & PI & Hpre & Hmax \\
\hline 83 & Graphistylis serrana & 1.6 & 0.03 & 0.16 & 0.03 & 0.10 & 41.0 & 41.0 \\
84 & Leandra quinquedentata & 1.6 & 0.03 & 0.16 & 0.03 & 0.10 & 45.0 & 49.0 \\
85 & Myrceugenia alpigea & 1.6 & 0.03 & 0.16 & 0.03 & 0.10 & 59.0 & 59.0 \\
86 & Oxypetalum sp. & 1.6 & 0.03 & 0.16 & 0.03 & 0.10 & 72.0 & 76.0 \\
87 & Tibouchina sp. 1 & 1.6 & 0.03 & 0.16 & 0.03 & 0.10 & 38.0 & 38.0 \\
88 & Weinmannia humilis & 1.6 & 0.03 & 0.16 & 0.03 & 0.10 & 58.0 & 67.0 \\
89 & Xyris piraquarae & 1.6 & 0.03 & 0.16 & 0.03 & 0.10 & 74.0 & 74.0 \\
90 & Baccharis nebularis & 1.6 & 0.02 & 0.16 & 0.01 & 0.09 & 70.0 & 70.0 \\
91 & Leandra cordigera & 1.6 & 0.02 & 0.16 & 0.01 & 0.09 & 18.0 & 21.0 \\
92 & Melastomataceae indet. 2 & 1.6 & 0.02 & 0.16 & 0.01 & 0.09 & 15.0 & 15.0 \\
93 & Pleopeltis hirsutissima & 1.6 & 0.02 & 0.16 & 0.01 & 0.09 & 34.0 & 34.0 \\
94 & Tibouchina sp. 2 & 1.6 & 0.02 & 0.16 & 0.01 & 0.09 & 17.0 & 17.0 \\
\hline & Total & 995.1 & 124.66 & 100 & 100 & 100 & & \\
\hline
\end{tabular}

plots within an absolute cover of $136 \mathrm{~m}^{2} \cdot 100^{-1} \mathrm{~m}^{2}$ (table 5). Chusquea mimosa comprised $25.7 \%$ of importance percentage. Shrubs and nanofanerophytes such as Croton mullerianus, Siphoneugen a reitzii and Vernonanthura montevidensis showed significant importance in this physiognomy with the stratum right below the the main species.

Altogether, Chusquea mimosa, Croton mullerianus, Cryptangium triquetrum, Siphoneugena reitzii and Vernonanthura montevidensis accounted for more than half the importance percentage and also comprised more than $70 \%$ of the cover percentage.

Serra do Ibitiraquire - Physiognomy: Machaerina austrobrasiliensis - We found 36 species in the 10 sampled plots (table 6) and a vegetation cover of $102 \mathrm{~m}^{2} \cdot 100^{-1} \mathrm{~m}^{2}$. Machaerina austrobrasiliensis accounted for more than a quarter the community dominance percentage as well as more than $40 \%$ of relative cover. Croton mullerianus showed high frequence. Even though Chusquea pinifolia was not very frequently found, it had a higher cover percentage in comparison with Xyris stenophylla, which had the same frequency. Cryptangium triquetrum, although very frequent, did not show high cover percentages. The five main species in this physiognomy comprised more than $80 \%$ of the relative cover and more than $60 \%$ of the importance percentage.

This physiognomy was observed more commonly at poor drainage sites with Xyris stenophylla, Xyris piraquarae and Baccharis angusticeps showing greater importance than in other vegetation physiognomy types.
Structure of upper montane grasslands in Serra da Igreja

Serra da Igreja - Physiognomy: shrubby - We found 50 species in the 27 sampled plots and a vegetation cover of $149 \mathrm{~m}^{2} \cdot 100^{-1} \mathrm{~m}^{2}$ (table 7). Croton mullerianus was the most important species occurring in all plots with nearly $40 \%$ of the relative cover. Cryptangium triquetrum was also important in almost all plots of this physiognomy. The four main species accounted for more than half the dominance percentage of the community. Despite having low cover in the physiognomy, Chusquea mimosa stood out as it showed the highest portions of the vertical strata with average height of $106.4 \mathrm{~cm}$ in comparison to plants higher than $2 \mathrm{~m}$ in height.

Other shrubby species such as Tibouchina dubia and Myrsine altomontana stood out by showing the same frequency, but the first species presented vegetation cover three times greater than the second one. Furthermore, Weinmannia humilis, Myrsine altomontana and Siphoneugena reitzii, also dominant in this community, can grow as trees over $6 \mathrm{~m}$ tall in adjacent upper montane rainforests. However, in upper montane grasslands they grow as nanophanerophytes to about $1 \mathrm{~m}$ in height.

Serra da Igreja - Physiognomy: grassy - 33 species were detected in the 14 sampled plots within a vegetation cover of $150.5 \mathrm{~m}^{2} .100^{-1} \mathrm{~m}^{2}$ (table 8 ). Similarly to the grasslands of Serra do Ibitiraquire, Cryptangium triquetrum was the most important species occurring in all plots and comprising more than $40 \%$ of the relative cover. Croton mullerianus 
Table 3. Phytosociological parameters of the grassy physiognomy of the upper montane grasslands in Serra do Ibitiraquire, $\mathrm{n}=64$. FA: absolute frequency (\%); Co: absolute cover (\%); FR: relative frequency (\%); CoR: relative cover (\%); PI: importance percentual (\%); Hpre: predominant height (cm); Hmax: maximum height (cm).

\begin{tabular}{|c|c|c|c|c|c|c|c|c|}
\hline & Species & FA & Co & FR & CoR & PI & Hpre & Hmax \\
\hline 1 & Cryptangium triquetrum & 51.6 & 29.00 & 6.31 & 26.54 & 16.42 & 54.6 & 67.3 \\
\hline 2 & Deschampsia caespitosa & 50.0 & 13.17 & 6.12 & 12.05 & 9.09 & 49.6 & 63.2 \\
\hline 3 & Eriochrysis holcoides & 40.6 & 11.19 & 4.97 & 10.24 & 7.60 & 41.5 & 53.0 \\
\hline 4 & Croton mullerianus & 34.4 & 5.92 & 4.21 & 5.42 & 4.81 & 40.0 & 48.6 \\
\hline 5 & Eryngium koehneanum & 31.3 & 5.77 & 3.82 & 5.28 & 4.55 & 42.3 & 50.3 \\
\hline 6 & Xyris stenophylla & 34.4 & 4.59 & 4.21 & 4.20 & 4.21 & 32.6 & 41.0 \\
\hline 7 & Chusquea pinifolia & 25.0 & 5.58 & 3.06 & 5.10 & 4.08 & 46.4 & 57.3 \\
\hline 8 & Vernonanthura montevidensis & 42.2 & 1.03 & 5.16 & 0.94 & 3.05 & 41.8 & 46.6 \\
\hline 9 & Eryngium scirpinum & 15.6 & 1.89 & 1.91 & 1.73 & 1.82 & 32.7 & 40.7 \\
\hline 10 & Hesperozygis nitida & 17.2 & 1.28 & 2.10 & 1.17 & 1.64 & 37.1 & 41.2 \\
\hline 11 & Machaerina austrobrasiliensis & 10.9 & 2.11 & 1.34 & 1.93 & 1.63 & 68.7 & 75.9 \\
\hline 12 & Nassella sellowiana & 10.9 & 1.78 & 1.34 & 1.63 & 1.48 & 56.0 & 80.9 \\
\hline 13 & Mimosa congestifolia & 15.6 & 1.13 & 1.91 & 1.03 & 1.47 & 52.9 & 58.2 \\
\hline 14 & Symphyopappus cuneatus & 14.1 & 1.30 & 1.72 & 1.19 & 1.45 & 50.8 & 58.3 \\
\hline 15 & Rhynchospora exaltata & 9.4 & 1.89 & 1.15 & 1.73 & 1.44 & 41.5 & 49.0 \\
\hline 16 & Chascolytrum calotheca & 14.1 & 1.25 & 1.72 & 1.14 & 1.43 & 65.3 & 74.3 \\
\hline 17 & Lobelia camporum & 18.8 & 0.45 & 2.29 & 0.41 & 1.35 & 25.0 & 27.3 \\
\hline 18 & Baccharis crispa & 18.8 & 0.42 & 2.29 & 0.39 & 1.34 & 39.0 & 42.3 \\
\hline 19 & Mimosa prionopus & 6.3 & 2.05 & 0.76 & 1.87 & 1.32 & 34.5 & 46.0 \\
\hline 20 & Achyrocline satureioides & 10.9 & 1.38 & 1.34 & 1.26 & 1.30 & 36.7 & 46.3 \\
\hline 21 & Lycopodium sp. & 14.1 & 0.92 & 1.72 & 0.84 & 1.28 & 21.9 & 26.9 \\
\hline 22 & Sisyrinchium vaginatum & 17.2 & 0.36 & 2.10 & 0.33 & 1.22 & 22.5 & 23.2 \\
\hline 23 & Rhynchospora sp. 1 & 12.5 & 0.98 & 1.53 & 0.90 & 1.22 & 37.1 & 53.6 \\
\hline 24 & Poaceae 13 & 15.6 & 0.48 & 1.91 & 0.44 & 1.18 & 20.5 & 22.7 \\
\hline 25 & Galianthe gertii & 10.9 & 0.52 & 1.34 & 0.47 & 0.91 & 30.1 & 33.3 \\
\hline 26 & Dichanthelium sabulorum & 10.9 & 0.48 & 1.34 & 0.44 & 0.89 & 33.3 & 58.0 \\
\hline 27 & Eriocaulon ligulatum & 10.9 & 0.47 & 1.34 & 0.43 & 0.88 & 19.4 & 31.6 \\
\hline 28 & Lycopodium thyoides & 6.3 & 0.94 & 0.76 & 0.86 & 0.81 & 28.5 & 38.5 \\
\hline 29 & Rhynchospora sp. 2 & 9.4 & 0.48 & 1.15 & 0.44 & 0.80 & 20.8 & 25.7 \\
\hline 30 & Chusquea mimosa & 7.8 & 0.69 & 0.96 & 0.63 & 0.79 & 58.0 & 72.0 \\
\hline 31 & Galium hypocarpium ssp. indecorum & 9.4 & 0.45 & 1.15 & 0.41 & 0.78 & 34.5 & 39.2 \\
\hline 32 & Leandra sp. & 9.4 & 0.22 & 1.15 & 0.20 & 0.67 & 18.5 & 19.8 \\
\hline 33 & Trixis lessingii & 7.8 & 0.34 & 0.96 & 0.31 & 0.64 & 17.2 & 18.8 \\
\hline 34 & Xyris piraquarae & 4.7 & 0.72 & 0.57 & 0.66 & 0.62 & 38.7 & 47.0 \\
\hline 35 & Lycopodium clavatum & 6.3 & 0.33 & 0.76 & 0.30 & 0.53 & 25.3 & 36.0 \\
\hline 36 & Dyckia reitzii & 6.3 & 0.31 & 0.76 & 0.29 & 0.53 & 20.8 & 22.5 \\
\hline 38 & Poaceae 11 & 6.3 & 0.19 & 0.76 & 0.17 & 0.47 & 46.3 & 60.8 \\
\hline 37 & Leguminosae indet. & 6.3 & 0.19 & 0.76 & 0.17 & 0.47 & 21.8 & 26.5 \\
\hline 40 & Tibouchina hospita & 6.3 & 0.14 & 0.76 & 0.13 & 0.45 & 29.3 & 29.5 \\
\hline 39 & Miconia sp. & 6.3 & 0.14 & 0.76 & 0.13 & 0.45 & 25.8 & 26.8 \\
\hline 41 & Lycopodium sp. 1 & 4.7 & 0.33 & 0.57 & 0.30 & 0.44 & 28.3 & 34.3 \\
\hline
\end{tabular}


Table 3 (continuation)

\begin{tabular}{|c|c|c|c|c|c|c|c|c|}
\hline & Species & FA & Co & FR & CoR & PI & Hpre & Hmax \\
\hline 42 & Utricularia reniformis & 4.7 & 0.33 & 0.57 & 0.30 & 0.44 & 22.3 & 28.0 \\
\hline 43 & $\begin{array}{l}\text { Gaylussacia brasiliensis var } \\
\text { brasiliensis }\end{array}$ & 6.3 & 0.11 & 0.76 & 0.10 & 0.43 & 27.0 & 30.8 \\
\hline 44 & Hypochaeris catharinensis & 6.3 & 0.11 & 0.76 & 0.10 & 0.43 & 20.5 & 31.5 \\
\hline 45 & Blechnum cordatum & 4.7 & 0.31 & 0.57 & 0.29 & 0.43 & 49.3 & 43.7 \\
\hline 46 & Baccharis angusticeps & 4.7 & 0.25 & 0.57 & 0.23 & 0.40 & 36.3 & 41.0 \\
\hline 47 & Sticherus sp. & 3.1 & 0.42 & 0.38 & 0.39 & 0.38 & 52.0 & 58.5 \\
\hline 48 & Campovassouria cruciata & 4.7 & 0.20 & 0.57 & 0.19 & 0.38 & 37.7 & 40.0 \\
\hline 49 & Alstroemeria amabilis & 4.7 & 0.14 & 0.57 & 0.13 & 0.35 & 32.3 & 37.7 \\
\hline 50 & Esterhazya splendida & 4.7 & 0.11 & 0.57 & 0.10 & 0.34 & 56.0 & 58.7 \\
\hline 52 & Symphyорарриs sp. 1 & 4.7 & 0.09 & 0.57 & 0.09 & 0.33 & 45.0 & 49.7 \\
\hline 51 & Gaylussacia arassatubensis & 4.7 & 0.09 & 0.57 & 0.09 & 0.33 & 12.3 & 13.0 \\
\hline 53 & Vriesea hoehneana & 1.6 & 0.47 & 0.19 & 0.43 & 0.31 & 30.0 & 41.0 \\
\hline 55 & Stevia claussenii & 4.7 & 0.05 & 0.57 & 0.04 & 0.31 & 25.7 & 25.7 \\
\hline 54 & Andropogon macrothrix & 4.7 & 0.05 & 0.57 & 0.04 & 0.31 & 16.3 & 16.3 \\
\hline 56 & Escallonia laevis & 3.1 & 0.23 & 0.38 & 0.21 & 0.30 & 58.0 & 65.5 \\
\hline 57 & Cyperaceae indet. 2 & 3.1 & 0.19 & 0.38 & 0.17 & 0.28 & 20.5 & 23.0 \\
\hline 58 & Cyperaceae indet. 1 & 3.1 & 0.16 & 0.38 & 0.14 & 0.26 & 16.0 & 16.0 \\
\hline 59 & Tibouchina dubia & 1.6 & 0.33 & 0.19 & 0.30 & 0.25 & 51.0 & 57.0 \\
\hline 60 & Leandra quinquedentata & 3.1 & 0.11 & 0.38 & 0.10 & 0.24 & 35.5 & 36.5 \\
\hline 61 & Galium sp. & 3.1 & 0.09 & 0.38 & 0.09 & 0.23 & 27.0 & 30.5 \\
\hline 62 & Gaultheria serrata var. organensis & 3.1 & 0.08 & 0.38 & 0.07 & 0.23 & 37.5 & 41.5 \\
\hline 63 & Poaceae 10 & 3.1 & 0.08 & 0.38 & 0.07 & 0.23 & 32.5 & 34.0 \\
\hline 64 & Poaceae 14 & 3.1 & 0.08 & 0.38 & 0.07 & 0.23 & 18.0 & 29.0 \\
\hline 65 & Tibouchina reitzii & 3.1 & 0.08 & 0.38 & 0.07 & 0.23 & 49.5 & 64.5 \\
\hline 66 & Phalocallis geniculata & 3.1 & 0.06 & 0.38 & 0.06 & 0.22 & 67.0 & 68.0 \\
\hline 67 & Ericaceae 02 & 3.1 & 0.06 & 0.38 & 0.06 & 0.22 & 40.5 & 43.5 \\
\hline 68 & Polygala campestris & 3.1 & 0.06 & 0.38 & 0.06 & 0.22 & 21.5 & 22.0 \\
\hline 69 & Axonopus sp. & 3.1 & 0.03 & 0.38 & 0.03 & 0.21 & 24.0 & 24.0 \\
\hline 70 & Drosera montana & 3.1 & 0.03 & 0.38 & 0.03 & 0.21 & 2.5 & 4.5 \\
\hline 71 & Fuchsia regia subsp. serrae & 3.1 & 0.03 & 0.38 & 0.03 & 0.21 & 22.5 & 22.5 \\
\hline 72 & Cyperaceae 6 & 1.6 & 0.22 & 0.19 & 0.20 & 0.20 & 47.0 & 71.0 \\
\hline 73 & Poaceae 15 & 1.6 & 0.20 & 0.19 & 0.19 & 0.19 & 59.0 & 64.0 \\
\hline 74 & Mimosa tucumensis & 1.6 & 0.17 & 0.19 & 0.16 & 0.17 & 23.0 & 31.0 \\
\hline 75 & Myrsine altomontana & 1.6 & 0.17 & 0.19 & 0.16 & 0.17 & 70.0 & 75.0 \\
\hline 76 & Handroanthus catarinesis & 1.6 & 0.14 & 0.19 & 0.13 & 0.16 & 65.0 & 65.0 \\
\hline 77 & Polygala subverticillata & 1.6 & 0.13 & 0.19 & 0.11 & 0.15 & 26.0 & 30.0 \\
\hline 78 & Oxypetalum sublanatum & 1.6 & 0.11 & 0.19 & 0.10 & 0.15 & 41.0 & 53.0 \\
\hline 79 & Myrcia hartwegiana & 1.6 & 0.09 & 0.19 & 0.09 & 0.14 & 45.0 & 47.0 \\
\hline 80 & Quesnelia imbricata & 1.6 & 0.09 & 0.19 & 0.09 & 0.14 & 38.0 & 38.0 \\
\hline & Weinmannia humilis & 1.6 & 0.08 & 0.19 & 0.07 & 0.13 & 30.0 & 45.0 \\
\hline 82 & Cyperaceae 2 & 1.6 & 0.06 & 0.19 & 0.06 & 0.12 & 45.0 & 47.0 \\
\hline 83 & Danthonia secundiflora & 1.6 & 0.06 & 0.19 & 0.06 & 0.12 & 55.0 & 68.0 \\
\hline
\end{tabular}


Table 3 (continuation)

\begin{tabular}{|c|c|c|c|c|c|c|c|c|}
\hline & Species & FA & $\mathrm{Co}$ & FR & CoR & PI & Hpre & Hmax \\
\hline 84 & Lycopodiaceae indet. & 1.6 & 0.05 & 0.19 & 0.04 & 0.12 & 1.0 & 1.0 \\
\hline 85 & Melastomataceae indet. 1 & 1.6 & 0.05 & 0.19 & 0.04 & 0.12 & 14.0 & 29.0 \\
\hline 86 & Piptocarpha densifolia & 1.6 & 0.05 & 0.19 & 0.04 & 0.12 & 47.0 & 47.0 \\
\hline 88 & Berberis laurina & 1.6 & 0.03 & 0.19 & 0.03 & 0.11 & 38.0 & 38.0 \\
\hline 87 & Viola cerasifolia & 1.6 & 0.05 & 0.19 & 0.04 & 0.12 & 14.0 & 17.0 \\
\hline 89 & Hesperozygis rhododon & 1.6 & 0.03 & 0.19 & 0.03 & 0.11 & 22.0 & 33.0 \\
\hline 90 & Leandra sulfurea & 1.6 & 0.03 & 0.19 & 0.03 & 0.11 & 18.0 & 50.0 \\
\hline 91 & NI 4 & 1.6 & 0.03 & 0.19 & 0.03 & 0.11 & 14.0 & 17.0 \\
\hline 92 & Poaceae 5 & 1.6 & 0.03 & 0.19 & 0.03 & 0.11 & 38.0 & 71.0 \\
\hline 93 & Apocynaceae 1 & 1.6 & 0.02 & 0.19 & 0.01 & 0.10 & 43.0 & 43.0 \\
\hline 94 & Baccharis nebularis & 1.6 & 0.02 & 0.19 & 0.01 & 0.10 & 73.0 & 73.0 \\
\hline 95 & Doryopteris crenulans & 1.6 & 0.02 & 0.19 & 0.01 & 0.10 & 28.0 & 28.0 \\
\hline 96 & Melastomataceae 2 & 1.6 & 0.02 & 0.19 & 0.01 & 0.10 & 13.0 & 13.0 \\
\hline 97 & NI 2 & 1.6 & 0.02 & 0.19 & 0.01 & 0.10 & 26.0 & 26.0 \\
\hline 98 & Scleria balansae & 1.6 & 0.02 & 0.19 & 0.01 & 0.10 & 13.0 & 18.0 \\
\hline 99 & Holocheilus brasiliensis & 1.6 & 0.02 & 0.19 & 0.01 & 0.10 & 30.0 & 30.0 \\
\hline & Total & 817.2 & 109.28 & 100 & 100 & 100 & & \\
\hline
\end{tabular}

Table 4. Phytosociological parameters of the Chusquea pinifolia physiognomy of the upper montane grasslands in Serra do Ibitiraquire, $\mathrm{n}=56$. FA: absolute frequency (\%); Co: absolute cover ( $\%)$; FR: relative frequency (\%); CoR: relative cover $(\%)$; PI: importance percentual (\%); Hpre: predominant height $(\mathrm{cm})$; Hmax: maximum height $(\mathrm{cm})$.

\begin{tabular}{llrrrrrc}
\hline Species & FA & \multicolumn{1}{c}{ Co } & FR & CoR & PI & Hpre & Hmax \\
\hline 1 Chusquea pinifolia & 100.0 & 54.07 & 9.62 & 43.26 & 26.44 & 78.4 & 99.4 \\
2 Croton mullerianus & 87.5 & 16.95 & 8.42 & 13.56 & 10.99 & 48.0 & 57.6 \\
3 Rhynchospora exaltata & 35.7 & 6.41 & 3.44 & 5.13 & 4.28 & 50.9 & 63.7 \\
4 Cryptangium triquetrum & 48.2 & 3.95 & 4.64 & 3.16 & 3.90 & 55.0 & 64.3 \\
5 Machaerina austrobrasiliensis & 35.7 & 4.82 & 3.44 & 3.86 & 3.65 & 67.2 & 80.1 \\
6 Alstroemeria amabilis & 39.3 & 1.71 & 3.78 & 1.37 & 2.58 & 42.6 & 49.6 \\
7 Deschampsia caespitosa & 33.9 & 1.30 & 3.26 & 1.04 & 2.15 & 53.6 & 65.9 \\
8 Gaylussacia brasiliensis var. brasiliensis & 30.4 & 1.55 & 2.92 & 1.24 & 2.08 & 47.7 & 58.0 \\
9 Xyris stenophylla & 26.8 & 1.75 & 2.58 & 1.40 & 1.99 & 35.1 & 42.3 \\
10 Baccharis crispa & 33.9 & 0.80 & 3.26 & 0.64 & 1.95 & 48.2 & 53.0 \\
11 Eriochrysis holcoides & 26.8 & 1.25 & 2.58 & 1.00 & 1.79 & 37.0 & 43.6 \\
12 Xyris piraquarae & 21.4 & 1.86 & 2.06 & 1.49 & 1.77 & 50.9 & 61.8 \\
13 Mimosa congestifolia & 19.6 & 1.66 & 1.89 & 1.33 & 1.61 & 64.0 & 68.6 \\
14 Leandra quinquedentata & 19.6 & 1.14 & 1.89 & 0.91 & 1.40 & 46.7 & 50.7 \\
15 Utricularia reniformis & 19.6 & 1.04 & 1.89 & 0.83 & 1.36 & 19.2 & 22.7 \\
16 Gaultheria serrata & 23.2 & 0.59 & 2.23 & 0.47 & 1.35 & 53.0 & 55.7 \\
17 Gaylussacia arassatubensis & 21.4 & 0.63 & 2.06 & 0.50 & 1.28 & 37.5 & 40.7 \\
18 Galium hypocarpium ssp. indecorum & 21.4 & 0.54 & 2.06 & 0.43 & 1.25 & 36.1 & 37.3 \\
19 Leandra sulfurea & 14.3 & 1.25 & 1.37 & 1.00 & 1.19 & 41.0 & 46.9 \\
20 Baccharis angusticeps & 12.5 & 1.34 & 1.20 & 1.07 & 1.14 & 58.0 & 59.1
\end{tabular}


Table 4 (continuation)

\begin{tabular}{|c|c|c|c|c|c|c|c|}
\hline Species & FA & $\mathrm{Co}$ & FR & CoR & PI & Hpre & Hmax \\
\hline 21 Hesperozygis nitida & 17.9 & 0.64 & 1.72 & 0.51 & 1.12 & 56.4 & 62.7 \\
\hline 22 Lycopodium thyoides & 16.1 & 0.86 & 1.55 & 0.69 & 1.12 & 28.2 & 38.2 \\
\hline 23 Myrsine altomontana & 16.1 & 0.73 & 1.55 & 0.59 & 1.07 & 76.7 & 82.9 \\
\hline 24 Sticherus sp. & 5.4 & 1.73 & 0.52 & 1.39 & 0.95 & 49.0 & 54.7 \\
\hline 25 Symphyopappus cuneatus & 12.5 & 0.80 & 1.20 & 0.64 & 0.92 & 62.1 & 69.0 \\
\hline 26 Tibouchina hospita & 14.3 & 0.48 & 1.37 & 0.39 & 0.88 & 42.9 & 47.3 \\
\hline 27 Rhynchospora sp. 1 & 7.1 & 1.25 & 0.69 & 1.00 & 0.84 & 41.8 & 50.8 \\
\hline 28 Eryngium koehneanum & 10.7 & 0.73 & 1.03 & 0.59 & 0.81 & 39.7 & 53.3 \\
\hline 29 Scleria balansae & 12.5 & 0.48 & 1.20 & 0.39 & 0.79 & 34.7 & 43.9 \\
\hline 30 Vernonanthura montevidensis & 12.5 & 0.34 & 1.20 & 0.27 & 0.74 & 55.9 & 60.0 \\
\hline 31 Melastomataceae indet. 1 & 12.5 & 0.30 & 1.20 & 0.24 & 0.72 & 23.6 & 24.7 \\
\hline 32 Mimosa tucumensis & 8.9 & 0.70 & 0.86 & 0.56 & 0.71 & 21.8 & 28.8 \\
\hline 33 Dyckia reitzii & 7.1 & 0.91 & 0.69 & 0.73 & 0.71 & 32.8 & 40.8 \\
\hline 34 Chascolytrum calotheca & 10.7 & 0.36 & 1.03 & 0.29 & 0.66 & 57.3 & 68.0 \\
\hline 35 Lycopodium sp. & 7.1 & 0.70 & 0.69 & 0.56 & 0.62 & 42.8 & 46.3 \\
\hline 36 Symplocos corymboclados & 7.1 & 0.63 & 0.69 & 0.50 & 0.59 & 56.0 & 56.8 \\
\hline 37 Trixis lessingii & 8.9 & 0.39 & 0.86 & 0.31 & 0.59 & 19.8 & 25.8 \\
\hline 38 Drimys angustifolia & 7.1 & 0.59 & 0.69 & 0.47 & 0.58 & 90.3 & 98.5 \\
\hline 39 Siphoneugena reitzii & 8.9 & 0.34 & 0.86 & 0.27 & 0.57 & 55.8 & 57.4 \\
\hline 40 Leptostelma catharinensis & 8.9 & 0.32 & 0.86 & 0.26 & 0.56 & 35.0 & 38.0 \\
\hline 41 Galianthe gertii & 7.1 & 0.46 & 0.69 & 0.37 & 0.53 & 36.8 & 43.3 \\
\hline 42 Eriocaulon ligulatum & 8.9 & 0.23 & 0.86 & 0.19 & 0.52 & 21.4 & 22.6 \\
\hline 43 Graphistylis serrana & 8.9 & 0.21 & 0.86 & 0.17 & 0.52 & 77.4 & 81.8 \\
\hline 44 Gaylussacia caratuvensis & 3.6 & 0.73 & 0.34 & 0.59 & 0.46 & 87.0 & 88.0 \\
\hline 45 Blechnum cordatum & 7.1 & 0.27 & 0.69 & 0.21 & 0.45 & 37.0 & 41.5 \\
\hline 46 Sisyrinchium vaginatum & 7.1 & 0.13 & 0.69 & 0.10 & 0.39 & 31.8 & 32.8 \\
\hline 47 Hypochaeris catharinensis & 5.4 & 0.27 & 0.52 & 0.21 & 0.36 & 18.7 & 39.0 \\
\hline 48 Dichanthelium sabulorum & 5.4 & 0.20 & 0.52 & 0.16 & 0.34 & 46.0 & 52.3 \\
\hline 49 Baccharis platypoda & 5.4 & 0.18 & 0.52 & 0.14 & 0.33 & 48.7 & 51.7 \\
\hline 50 Poaceae 5 & 5.4 & 0.18 & 0.52 & 0.14 & 0.33 & 15.3 & 15.3 \\
\hline 51 Baccharis nebularis & 5.4 & 0.14 & 0.52 & 0.11 & 0.31 & 62.0 & 66.3 \\
\hline 52 Miconia ramboi & 5.4 & 0.07 & 0.52 & 0.06 & 0.29 & 34.7 & 35.7 \\
\hline 53 Weinmannia humilis & 1.8 & 0.50 & 0.17 & 0.40 & 0.29 & 98.0 & 108.0 \\
\hline 54 Ilex chamaedryfolia & 3.6 & 0.25 & 0.34 & 0.20 & 0.27 & 86.5 & 99.0 \\
\hline 55 Mimosa prionopus & 3.6 & 0.21 & 0.34 & 0.17 & 0.26 & 47.0 & 50.0 \\
\hline 56 Doryopteris crenulans & 3.6 & 0.20 & 0.34 & 0.16 & 0.25 & 31.0 & 44.0 \\
\hline 57 Achyrocline satureioides & 3.6 & 0.18 & 0.34 & 0.14 & 0.24 & 59.5 & 66.5 \\
\hline 58 Mimosa eurystegia & 3.6 & 0.18 & 0.34 & 0.14 & 0.24 & 40.5 & 43.0 \\
\hline 59 Escallonia laevis & 1.8 & 0.38 & 0.17 & 0.30 & 0.24 & 100.0 & 108.0 \\
\hline 60 Tibouchina dubia & 3.6 & 0.13 & 0.34 & 0.10 & 0.22 & 33.5 & 36.5 \\
\hline 61 Lycopodium sp. 1 & 1.8 & 0.32 & 0.17 & 0.26 & 0.21 & 20.0 & 26.0 \\
\hline 62 Gaultheria sp. 1 & 3.6 & 0.09 & 0.34 & 0.07 & 0.21 & 49.5 & 49.5 \\
\hline
\end{tabular}


Table 4 (continuation)

\begin{tabular}{lccccccc}
\hline Species & FA & Co & FR & CoR & PI & Hpre & Hmax \\
\hline 63 Ericaceae indet. 1 & 3.6 & 0.09 & 0.34 & 0.07 & 0.21 & 36.5 & 40.5 \\
64 Leandra sp. & 3.6 & 0.07 & 0.34 & 0.06 & 0.20 & 37.5 & 41.5 \\
65 Piptocarpha densifolia & 1.8 & 0.29 & 0.17 & 0.23 & 0.20 & 37.0 & 82.0 \\
66 Gaultheria serrata var. organensis & 3.6 & 0.05 & 0.34 & 0.04 & 0.19 & 63.5 & 63.5 \\
67 Phalocallis geniculata & 3.6 & 0.05 & 0.34 & 0.04 & 0.19 & 55.0 & 62.0 \\
68 Melastomataceae indet 2 & 3.6 & 0.05 & 0.34 & 0.04 & 0.19 & 14.0 & 14.0 \\
69 Dicranopteris nervosa & 1.8 & 0.20 & 0.17 & 0.16 & 0.16 & 32.0 & 32.0 \\
70 Sticherus pruinosus & 1.8 & 0.11 & 0.17 & 0.09 & 0.13 & 34.0 & 37.0 \\
71 Polygala campestris & 1.8 & 0.11 & 0.17 & 0.09 & 0.13 & 29.0 & 29.0 \\
72 Lycopodium sp. 2 & 1.8 & 0.09 & 0.17 & 0.07 & 0.12 & 40.0 & 58.0 \\
73 Ericaceae indet. 2 & 1.8 & 0.07 & 0.17 & 0.06 & 0.11 & 52.0 & 54.0 \\
74 Fabaceae & 1.8 & 0.07 & 0.17 & 0.06 & 0.11 & 24.0 & 28.0 \\
75 Leandra cordigera & 1.8 & 0.07 & 0.17 & 0.06 & 0.11 & 15.0 & 22.0 \\
76 Andropogon macrothrix & 1.8 & 0.05 & 0.17 & 0.04 & 0.11 & 25.0 & 41.0 \\
77 Smilax campestris & 1.8 & 0.04 & 0.17 & 0.03 & 0.10 & 53.0 & 53.0 \\
78 Campovassouria cruciata & 1.8 & 0.04 & 0.17 & 0.03 & 0.10 & 29.0 & 29.0 \\
79 Galium sellowianum & 1.8 & 0.04 & 0.17 & 0.03 & 0.10 & 15.0 & 17.0 \\
80 Drosera montana & 1.8 & 0.04 & 0.17 & 0.03 & 0.10 & 3.0 & 3.0 \\
81 Salvia sp. & 1.8 & 0.02 & 0.17 & 0.01 & 0.09 & 47.0 & 47.0 \\
82 Paspalum polyphyllum & 1.8 & 0.02 & 0.17 & 0.01 & 0.09 & 37.0 & 67.0 \\
83 Ilex microdonta & 1.8 & 0.02 & 0.17 & 0.01 & 0.09 & 27.0 & 27.0 \\
84 Lobelia camporum & 1.8 & 0.02 & 0.17 & 0.01 & 0.09 & 22.0 & 22.0 \\
85 Galium sp. & 1.8 & 0.02 & 0.17 & 0.01 & 0.09 & 14.0 & 14.0 \\
\hline Total & 1039.3 & 125.00 & 100 & 100 & 100 & & \\
\hline & & & & & & \\
\hline
\end{tabular}

Table 5. Phytosociological parameters of the Chusquea mimosa physiognomy of the upper montane grasslands in Serra do Ibitiraquire, $\mathrm{n}=9$. FA: absolute frequency ( $\%)$; Co: absolute cover (\%); FR: relative frequency (\%); CoR: relative cover (\%); PI: importance percentual (\%); Hpre: predominant height (cm); Hmax: maximum height (cm).

\begin{tabular}{llrrrrrrr}
\hline Species & FA & \multicolumn{1}{c}{ Co } & FR & \multicolumn{1}{c}{ CoR } & PI & Hpre & Hmax \\
\hline 1 Chusquea mimosa & 100.0 & 56.67 & 9.89 & 41.56 & 25.73 & 122.8 & 166.9 \\
2 Croton mullerianus & 66.7 & 23.56 & 6.59 & 17.28 & 11.94 & 58.0 & 72.5 \\
3 Cryptangium triquetrum & 44.4 & 9.22 & 4.40 & 6.76 & 5.58 & 64.3 & 76.0 \\
4 & Siphoneugena reitzii & 44.4 & 5.33 & 4.40 & 3.91 & 4.15 & 76.3 & 88.8 \\
5 & Vernonanthura montevidensis & 55.6 & 1.78 & 5.49 & 1.30 & 3.40 & 67.2 & 77.0 \\
6 Sticherus sp. & 33.3 & 3.56 & 3.30 & 2.61 & 2.95 & 47.0 & 56.0 \\
7 & Eryngium koehneanum & 33.3 & 2.89 & 3.30 & 2.12 & 2.71 & 34.3 & 37.0 \\
8 & Baccharis crispa & 44.4 & 1.33 & 4.40 & 0.98 & 2.69 & 51.8 & 57.5 \\
9 Xyris stenophylla & 22.2 & 3.00 & 2.20 & 2.20 & 2.20 & 35.0 & 46.0 \\
10 & Pentacalia desiderabilis & 11.1 & 4.33 & 1.10 & 3.18 & 2.14 & 65.0 & 85.0 \\
11 & Smilax campestris & 33.3 & 0.78 & 3.30 & 0.57 & 1.93 & 39.7 & 61.7 \\
12 & Dichanthelium sabulorum & 22.2 & 2.11 & 2.20 & 1.55 & 1.87 & 26.0 & 47.0 \\
13 Utricularia reniformis & 22.2 & 1.22 & 2.20 & 0.90 & 1.55 & 21.5 & 25.0
\end{tabular}


Table 5 (continuation)

\begin{tabular}{|c|c|c|c|c|c|c|c|c|}
\hline & Species & FA & Co & FR & CoR & PI & Hpre & Hmax \\
\hline 14 & Leandra sulfurea & 22.2 & 1.11 & 2.20 & 0.81 & 1.51 & 38.0 & 42.5 \\
\hline 15 & Vriesea hoehneana & 11.1 & 2.56 & 1.10 & 1.87 & 1.49 & 75.0 & 82.0 \\
\hline 16 & Galium hypocarpium ssp. indecorum & 22.2 & 1.00 & 2.20 & 0.73 & 1.47 & 38.5 & 46.5 \\
\hline 17 & Lycopodium sp. & 22.2 & 1.00 & 2.20 & 0.73 & 1.47 & 36.0 & 40.5 \\
\hline 18 & Mimosa prionopus & 11.1 & 2.22 & 1.10 & 1.63 & 1.36 & 13.0 & 29.0 \\
\hline 19 & Hydrocotyle quinqueloba & 22.2 & 0.67 & 2.20 & 0.49 & 1.34 & 37.5 & 39.5 \\
\hline 20 & Phalocallis geniculata & 22.2 & 0.56 & 2.20 & 0.41 & 1.30 & 52.0 & 57.5 \\
\hline 21 & Alstroemeria amabilis & 22.2 & 0.56 & 2.20 & 0.41 & 1.30 & 49.0 & 52.5 \\
\hline 22 & Myrsine altomontana & 22.2 & 0.56 & 2.20 & 0.41 & 1.30 & 49.0 & 52.5 \\
\hline 23 & Leandra sp. & 22.2 & 0.33 & 2.20 & 0.24 & 1.22 & 29.0 & 30.0 \\
\hline 24 & Esterhazya splendida & 11.1 & 1.78 & 1.10 & 1.30 & 1.20 & 78.0 & 85.0 \\
\hline 25 & Deschampsia caespitosa & 22.2 & 0.22 & 2.20 & 0.16 & 1.18 & 73.5 & 77.5 \\
\hline 26 & Hesperozygis nitida & 22.2 & 0.22 & 2.20 & 0.16 & 1.18 & 78.5 & 78.5 \\
\hline 27 & Sticherus pruinosus & 11.1 & 1.11 & 1.10 & 0.81 & 0.96 & 46.0 & 67.0 \\
\hline 28 & Poaceae 16 & 11.1 & 0.89 & 1.10 & 0.65 & 0.88 & 53.0 & 61.0 \\
\hline 29 & Rhynchospora sp. 2 & 11.1 & 0.89 & 1.10 & 0.65 & 0.88 & 28.0 & 28.0 \\
\hline 30 & Symphyopappus cuneatus & 11.1 & 0.78 & 1.10 & 0.57 & 0.83 & 67.0 & 71.0 \\
\hline 31 & Gaylussacia brasiliensis var brasiliensis & 11.1 & 0.44 & 1.10 & 0.33 & 0.71 & 40.0 & 45.0 \\
\hline 32 & Machaerina austrobrasiliensis & 11.1 & 0.44 & 1.10 & 0.33 & 0.71 & 45.0 & 65.0 \\
\hline 33 & Poaceae 5 & 11.1 & 0.44 & 1.10 & 0.33 & 0.71 & 16.0 & 16.0 \\
\hline 34 & Rhynchospora exaltata & 11.1 & 0.44 & 1.10 & 0.33 & 0.71 & 90.0 & 110.0 \\
\hline 35 & Baccharis nebularis & 11.1 & 0.33 & 1.10 & 0.24 & 0.67 & 52.0 & 57.0 \\
\hline 36 & Xyris piraquarae & 11.1 & 0.33 & 1.10 & 0.24 & 0.67 & 75.0 & 76.0 \\
\hline 37 & Gaultheria serrata & 11.1 & 0.22 & 1.10 & 0.16 & 0.63 & 31.0 & 31.0 \\
\hline 38 & Gaultheria sp. 1 & 11.1 & 0.22 & 1.10 & 0.16 & 0.63 & 35.0 & 42.0 \\
\hline 39 & Lycopodium clavatum & 11.1 & 0.22 & 1.10 & 0.16 & 0.63 & 35.0 & 35.0 \\
\hline 40 & Lycopodium thyoides & 11.1 & 0.22 & 1.10 & 0.16 & 0.63 & 20.0 & 21.0 \\
\hline 41 & Poaceae 14 & 11.1 & 0.22 & 1.10 & 0.16 & 0.63 & 56.0 & 56.0 \\
\hline 42 & Fuchsia regia subsp. serrae & 11.1 & 0.11 & 1.10 & 0.08 & 0.59 & 103.0 & 103.0 \\
\hline 43 & Galianthe gertii & 11.1 & 0.11 & 1.10 & 0.08 & 0.59 & 44.0 & 44.0 \\
\hline 44 & Melastomataceae 1 & 11.1 & 0.11 & 1.10 & 0.08 & 0.59 & 25.0 & 25.0 \\
\hline 45 & Myrciaria tenella & 11.1 & 0.11 & 1.10 & 0.08 & 0.59 & 44.0 & 44.0 \\
\hline & Tibouchina hospita & 11.1 & 0.11 & 1.10 & 0.08 & 0.59 & 33.0 & 33.0 \\
\hline & Total & 1011.1 & 136.33 & 100 & 100 & 100 & & \\
\hline
\end{tabular}

was also found in all the plots, but showed lower cover. Only these two species along with Tibouchina dubia (shrubby), accounted for $50 \%$ of the importance percentage and more than $70 \%$ of the community relative cover.

The seven main species, which comprised more than $80 \%$ of the relative cover $\left(125 \mathrm{~m}^{2} .100^{-1} \mathrm{~m}^{2}\right)$, showed average height between 45 and $65 \mathrm{~cm}$, clearly indicating the large overlap in this portion of the community vertical stratification.
Structure of upper montane grasslands in Serra da Prata

Serra da Prata - Physiognomy: shrubby - We found 36 species in the 15 sampled plots within an absolute cover of $121 \mathrm{~m}^{2} \cdot 100^{-1} \mathrm{~m}^{2}$ (table 9). Croton mullerianus displayed the highest percentage of dominance, being present in all plots and comprising almost a third of the community relative cover. Cryptangium triquetrum, Machaerina austrobrasiliensis and Tibouchina dubia also stood out with very close percentages of 
dominance in this physiognomy. These four species accounted for almost half the dominance percentage and more than $60 \%$ of the cover percentage.

Solely six species were found in at least two thirds of the plots. Half of the detected species occurred in only one or two plots, and these species comprised just $10 \%$ of the community importance percentage.

Serra da Prata - Physiognomy: grassy - We found 30 species in the seven sampled plots within an absolute cover of $124.7 \mathrm{~m}^{2} \cdot 100^{-1} \mathrm{~m}^{2}$ (table 10 ). Once again,

Table 6. Phytosociological parameters of the Machaerina austrobrasiliensis physiognomy of the upper montane grasslands in Serra do Ibitiraquire, $\mathrm{n}=10$. FA: absolute frequency (\%); Co: absolute cover (\%); FR: relative frequency (\%); CoR: relative cover (\%); PI: importance percentual (\%); Hpre: predominant height (cm); Hmax: maximum height (cm).

\begin{tabular}{|c|c|c|c|c|c|c|c|c|}
\hline & Species & FA & Co & FR & $\mathrm{CoR}$ & PI & Hpre & Hmax \\
\hline 1 & Machaerina austrobrasiliensis & 100 & 41.20 & 10.87 & 40.39 & 25.63 & 60.9 & 76.1 \\
\hline 2 & Croton mullerianus & 90 & 16.50 & 9.78 & 16.18 & 12.98 & 36.8 & 44.8 \\
\hline 3 & Chusquea pinifolia & 60 & 11.00 & 6.52 & 10.78 & 8.65 & 46.8 & 56.8 \\
\hline 4 & Xyris stenophylla & 60 & 7.80 & 6.52 & 7.65 & 7.08 & 28.7 & 35.8 \\
\hline 5 & Cryptangium triquetrum & 80 & 5.50 & 8.70 & 5.39 & 7.04 & 52.0 & 66.3 \\
\hline 6 & Eriochrysis holcoides & 40 & 2.60 & 4.35 & 2.55 & 3.45 & 34.0 & 42.0 \\
\hline 7 & Deschampsia caespitosa & 50 & 0.80 & 5.43 & 0.78 & 3.11 & 48.0 & 52.4 \\
\hline 8 & Baccharis angusticeps & 30 & 1.50 & 3.26 & 1.47 & 2.37 & 38.0 & 41.0 \\
\hline 9 & Xyris piraquarae & 30 & 0.80 & 3.26 & 0.78 & 2.02 & 57.0 & 62.7 \\
\hline 10 & Rhynchospora exaltata & 20 & 1.90 & 2.17 & 1.86 & 2.02 & 36.5 & 51.5 \\
\hline 11 & Galianthe gertii & 20 & 0.90 & 2.17 & 0.88 & 1.53 & 26.0 & 30.0 \\
\hline 12 & Chusquea mimosa & 10 & 2.00 & 1.09 & 1.96 & 1.52 & 85.0 & 117.0 \\
\hline 13 & Leandra sp. & 20 & 0.80 & 2.17 & 0.78 & 1.48 & 28.0 & 31.5 \\
\hline 14 & Dyckia reitzii & 20 & 0.50 & 2.17 & 0.49 & 1.33 & 19.0 & 20.5 \\
\hline 15 & Eriocaulon ligulatum & 20 & 0.50 & 2.17 & 0.49 & 1.33 & 26.5 & 28.5 \\
\hline 16 & Gaylussacia brasiliensis var brasiliensis & 20 & 0.50 & 2.17 & 0.49 & 1.33 & 26.5 & 29.0 \\
\hline 17 & Utricularia reniformis & 20 & 0.50 & 2.17 & 0.49 & 1.33 & 31.0 & 43.0 \\
\hline 18 & Melastomataceae indet. 1 & 20 & 0.40 & 2.17 & 0.39 & 1.28 & 14.0 & 15.5 \\
\hline 19 & Rhynchospora sp. 1 & 20 & 0.40 & 2.17 & 0.39 & 1.28 & 39.0 & 42.5 \\
\hline 20 & Baccharis crispa & 20 & 0.30 & 2.17 & 0.29 & 1.23 & 39.5 & 39.5 \\
\hline 21 & Leandra cordigera & 20 & 0.20 & 2.17 & 0.20 & 1.18 & 18.5 & 18.5 \\
\hline 22 & Leandra sulfurea & 10 & 1.00 & 1.09 & 0.98 & 1.03 & 49.0 & 58.0 \\
\hline 23 & Symphyopappus cuneatus & 10 & 0.80 & 1.09 & 0.78 & 0.94 & 42.0 & 57.0 \\
\hline 24 & Poaceae 05 & 10 & 0.50 & 1.09 & 0.49 & 0.79 & 9.0 & 9.0 \\
\hline 25 & Quesnelia imbricata & 10 & 0.50 & 1.09 & 0.49 & 0.79 & 40.0 & 44.0 \\
\hline 26 & Gaylussacia arassatubensis & 10 & 0.40 & 1.09 & 0.39 & 0.74 & 20.0 & 23.0 \\
\hline 27 & Mimosa congestifolia & 10 & 0.40 & 1.09 & 0.39 & 0.74 & 53.0 & 59.0 \\
\hline 28 & Mimosa tucumensis & 10 & 0.40 & 1.09 & 0.39 & 0.74 & 23.0 & 30.0 \\
\hline 29 & Eryngium koehneanum & 10 & 0.30 & 1.09 & 0.29 & 0.69 & 37.0 & 39.0 \\
\hline 30 & Sticherus sp. & 10 & 0.30 & 1.09 & 0.29 & 0.69 & 44.0 & 44.0 \\
\hline 31 & Scleria balansae & 10 & 0.20 & 1.09 & 0.20 & 0.64 & 10.0 & 12.0 \\
\hline 32 & Tibouchina hospita & 10 & 0.20 & 1.09 & 0.20 & 0.64 & 17.0 & 23.0 \\
\hline 33 & Galium hypocarpium ssp. indecorum & 10 & 0.10 & 1.09 & 0.10 & 0.59 & 15.0 & 15.0 \\
\hline 34 & Chascolytrum calotheca & 10 & 0.10 & 1.09 & 0.10 & 0.59 & 55.0 & 55.0 \\
\hline 35 & Sisyrinchium vaginatum & 10 & 0.10 & 1.09 & 0.10 & 0.59 & 40.0 & 40.0 \\
\hline \multirow[t]{2}{*}{36} & Trixis lessingii & 10 & 0.10 & 1.09 & 0.10 & 0.59 & 20.0 & 20.0 \\
\hline & Total & 920 & 102 & 100 & 100 & 100 & & \\
\hline
\end{tabular}


Cryptangium triquetrum was the most important species in this physiognomy. However, Croton mullerianus also stood out, being present in all plots and reaching the second highest percentage of importance. We highlight the importance of species frequently found in poor drainage environments such as Machaerina austrobrasiliensis, Xyris stenophylla and Xyris piraquarae.

In the above strata we found shrubs and nanophanerophytes such as Clethra uleana, Siphoneugena reitzii, Myrcia hartwegiana and Hesperozygis rhododon, whereas graminoids formed a dense vegetative layer of nearly $50 \mathrm{~cm}$ from the ground. From the most important species, only Machaerina austrobrasiliensis showed predominant height (frequently above $60 \mathrm{~cm}$ ).

Nearly two thirds of the species (19) were detected in only one or two sampled plots.

Structure of upper montane grasslands in Serra da Farinha Seca

Serra da Farinha Seca - Physiognomy: shrubby 42 species were found in the 10 sampled plots within a vegetation cover of $134.7 \mathrm{~m}^{2} .100^{-1} \mathrm{~m}^{2}$ (table 11). Mimosa congestifolia comprised the highest percentage of importance, and stood out based on its cover percentage. It accounted for nearly a quarter of the community relative cover. Cryptangium triquetrum, Sticherus sp., Chusquea pinifolia along with a non-identified Poaceae showed importance as well. Other shrubby species ranked only in the sixth and seventh position, namely: Siphoneugena reitzii and Myrcia hartwegiana. The seven species mentioned above comprised $54 \%$ of the importance percentage and almost $70 \%$ of the relative cover.

Chusquea pinifolia is quite important in this physiognomy based upon its frequency in the above strata and also for having all prevailing and maximum heights among the main species.

Serra da Farinha Seca - Physiognomy: grassy 53 species were found in the 22 sampled plots within an absolute cover of $148 \mathrm{~m}^{2} \cdot 100^{-1} \mathrm{~m}^{2}$ (2). Cryptangium triquetrum showed great importance in this physiognomy as it was found in all the plots, comprising $49 \%$ of the relative cover by dominating the upper strata of the community. Its average height was higher than the other eight important species, by creating a typical "graminoid"carpet.

Siphoneugena reitzii and Tibouchina dubia also stood out as shrubby species. However, despite their high frequency, they showed low cover values since each species accounted for solely $4 \%$ of the relative cover.

As a matter of fact, only these three species were detected in more than a third of the sampled plots. We also observed that 36 , or more than two thirds of the species, showed relative cover values lower than $1 \%$, which represents only $11 \%$ of the overall community cover.

Serra da Farinha Seca - Physiognomy: Chusquea pinifolia - Twenty-nine species were found in five sampled plots within an absolute cover of $134.6 \mathrm{~m}^{2} \cdot 100^{-1} \mathrm{~m}^{2}$ (table 13). Chusquea pinifolia showed $30.7 \%$ of importance percentage, which represents more than half the relative cover. It was also the dominant species in the upper strata, reaching prevailing heights greater than the other species, with the exception of a non-identified Poaceae.

In the stretches where $C$. pinifolia was the most dominant, several other important shrubs were also often found occupying spaces under Chusquea pinifolia in the vertical strata, especially Mimosa congestifolia, Siphoneugena reitzii, Baccharis nebularis, Symplocos corymboclados and Clethra scabra. The latter was exclusively found in Serra da Farinha Seca. The occurrence of Clethra uleana is common in upper montane grasslands, while Clethra scabra is more common in montane forests at altitudes $<1200$ m asl. However, in Serra da Farinha Seca both species share the same environment, but C. scabra seems to occur only in physiognomies dominated by Chusquea pinifolia. Falkenberg (2003) had already reported the overlapping area of occurrence of these two species in upper montane cloud forest and rupicolous vegetation in the region of Aparados da Serra Geral.

Structure of upper montane grasslands in Serra Gigante

Serra Gigante - Physiognomy: grassy - 23 species were found in the 12 sampled plots within an absolute cover of $112.4 \mathrm{~m}^{2} \cdot 100^{-1} \mathrm{~m}^{2}$ (table 14). Even though this montane range is found to be considerably lower with small-scale grasslands in comparison to the others studied herewith, it presents a typical physiognomy of this formation, but also includes some species which were not sampled in the other upper montane grasslands. Garcia \& Pirani (2005) also classified as "altitude grassland" or "high altitude grassland" the grassland vegetation in lower altitude mountain 
Table 7. Phytosociological parameters of the shrubby physiognomy of the upper montane grasslands in Serra da Igreja, $\mathrm{n}=27$. FA: absolute frequency $(\%)$; Co: absolute cover $(\%)$; FR: relative frequency (\%); CoR: relative cover $(\%)$; PI: importance percentual (\%); Hpre: predominant height (cm); Hmax: maximum height (cm).

\begin{tabular}{|c|c|c|c|c|c|c|c|c|}
\hline & Species & FA & Co & FR & CoR & PI & Hpre & Hmax \\
\hline 1 & Croton mullerianus & 100.0 & 59.19 & 9.57 & 39.63 & 24.60 & 67.6 & 78.1 \\
\hline 2 & Cryptangium triquetrum & 96.3 & 27.78 & 9.22 & 18.60 & 13.91 & 69.7 & 86.1 \\
\hline 3 & Sticherus sp. & 66.7 & 13.11 & 6.38 & 8.78 & 7.58 & 56.3 & 62.6 \\
\hline 4 & Tibouchina dubia & 74.1 & 8.70 & 7.09 & 5.83 & 6.46 & 50.0 & 60.7 \\
\hline 5 & Myrsine altomontana & 74.1 & 2.74 & 7.09 & 1.84 & 4.46 & 53.4 & 61.3 \\
\hline 6 & Alstroemeria amabilis & 55.6 & 1.93 & 5.32 & 1.29 & 3.30 & 48.2 & 59.3 \\
\hline 7 & Xyris stenophylla & 33.3 & 4.70 & 3.19 & 3.15 & 3.17 & 40.0 & 48.6 \\
\hline 8 & Leandra cordigera & 40.7 & 1.93 & 3.90 & 1.29 & 2.60 & 53.1 & 59.5 \\
\hline 9 & Poaceae 2 & 33.3 & 2.37 & 3.19 & 1.59 & 2.39 & 58.3 & 68.0 \\
\hline 10 & Blechnum cordatum & 37.0 & 1.26 & 3.55 & 0.84 & 2.19 & 38.0 & 47.7 \\
\hline 11 & Siphoneugena reitzii & 37.0 & 1.15 & 3.55 & 0.77 & 2.16 & 60.2 & 62.6 \\
\hline 12 & Weinmannia humilis & 22.2 & 2.44 & 2.13 & 1.64 & 1.88 & 82.8 & 85.3 \\
\hline 13 & Galium hypocarpium ssp. indecorum & 25.9 & 1.70 & 2.48 & 1.14 & 1.81 & 38.4 & 44.9 \\
\hline 14 & Chusquea mimosa & 18.5 & 2.48 & 1.77 & 1.66 & 1.72 & 106.4 & 140.2 \\
\hline 15 & Gaylussacia brasiliensis var brasiliensis & 29.6 & 0.48 & 2.84 & 0.32 & 1.58 & 29.0 & 33.0 \\
\hline 16 & Hesperozygis rhododon & 14.8 & 2.00 & 1.42 & 1.34 & 1.38 & 60.3 & 66.0 \\
\hline 17 & Handroanthus catarinesis & 22.2 & 0.85 & 2.13 & 0.57 & 1.35 & 71.3 & 78.5 \\
\hline 18 & Dichanthelium sabulorum & 18.5 & 1.22 & 1.77 & 0.82 & 1.30 & 46.0 & 44.8 \\
\hline 19 & Poaceae 3 & 22.2 & 0.48 & 2.13 & 0.32 & 1.23 & 42.2 & 47.5 \\
\hline 20 & Vriesea platynema var. variegata & 7.4 & 2.52 & 0.71 & 1.69 & 1.20 & 61.0 & 63.0 \\
\hline 21 & Eriocaulon ligulatum & 14.8 & 1.33 & 1.42 & 0.89 & 1.16 & 27.0 & 40.8 \\
\hline 22 & Gaultheria serrata var. organensis & 18.5 & 0.74 & 1.77 & 0.50 & 1.13 & 48.4 & 51.8 \\
\hline 23 & Baccharis crispa & 18.5 & 0.70 & 1.77 & 0.47 & 1.12 & 56.6 & 63.4 \\
\hline 24 & Clethra uleana & 14.8 & 0.67 & 1.42 & 0.45 & 0.93 & 42.8 & 63.0 \\
\hline 25 & Myrcia pulchra & 7.4 & 1.56 & 0.71 & 1.04 & 0.88 & 58.0 & 64.0 \\
\hline 26 & Vernonanthura montevidensis & 11.1 & 0.59 & 1.06 & 0.40 & 0.73 & 61.3 & 66.7 \\
\hline 27 & Baccharis angusticeps & 11.1 & 0.37 & 1.06 & 0.25 & 0.66 & 50.0 & 61.0 \\
\hline 28 & Utricularia reniformis & 11.1 & 0.33 & 1.06 & 0.22 & 0.64 & 23.0 & 30.0 \\
\hline 29 & Sticherus pruinosus & 7.4 & 0.56 & 0.71 & 0.37 & 0.54 & 52.5 & 56.0 \\
\hline 30 & Graphistylis serrana & 7.4 & 0.26 & 0.71 & 0.17 & 0.44 & 47.5 & 53.0 \\
\hline 31 & Smilax campestris & 7.4 & 0.26 & 0.71 & 0.17 & 0.44 & 52.5 & 55.0 \\
\hline 32 & Myrciaria tenella & 7.4 & 0.19 & 0.71 & 0.12 & 0.42 & 39.5 & 42.0 \\
\hline 33 & Apocynaceae 1 & 7.4 & 0.15 & 0.71 & 0.10 & 0.40 & 45.0 & 49.0 \\
\hline 34 & Myrcia hartwegiana & 7.4 & 0.11 & 0.71 & 0.07 & 0.39 & 61.0 & 61.0 \\
\hline & Holocheilus brasiliensis & 7.4 & 0.11 & 0.71 & 0.07 & 0.39 & 19.5 & 19.5 \\
\hline 36 & Vriesea hoehneana & 3.7 & 0.56 & 0.35 & 0.37 & 0.36 & 42.0 & 50.0 \\
\hline & Coccocypselum condalia & 3.7 & 0.52 & 0.35 & 0.35 & 0.35 & 8.0 & 14.0 \\
\hline & Blechnum sp. 2 & 3.7 & 0.41 & 0.35 & 0.27 & 0.31 & 73.0 & 85.0 \\
\hline & Drimys angustifolia & 3.7 & 0.11 & 0.35 & 0.07 & 0.21 & 63.0 & 71.0 \\
\hline 40 & Ericaceae 4 & 3.7 & 0.11 & 0.35 & 0.07 & 0.21 & 47.0 & 53.0 \\
\hline
\end{tabular}


Table 7 (continuation)

\begin{tabular}{|c|c|c|c|c|c|c|c|}
\hline Species & FA & $\mathrm{Co}$ & FR & CoR & PI & Hpre & Hmax \\
\hline 41 Ocotea tristis & 3.7 & 0.11 & 0.35 & 0.07 & 0.21 & 30.0 & 54.0 \\
\hline 42 Symplocos corymboclados & 3.7 & 0.11 & 0.35 & 0.07 & 0.21 & 33.0 & 41.0 \\
\hline 43 Danthonia sp. & 3.7 & 0.07 & 0.35 & 0.05 & 0.20 & 17.0 & 17.0 \\
\hline 44 Mikania clematidifolia & 3.7 & 0.07 & 0.35 & 0.05 & 0.20 & 66.0 & 66.0 \\
\hline 45 Symplocos corymboclados & 3.7 & 0.07 & 0.35 & 0.05 & 0.20 & 41.0 & 60.0 \\
\hline 46 Ternstroemia brasiliensis & 3.7 & 0.07 & 0.35 & 0.05 & 0.20 & 69.0 & 69.0 \\
\hline 47 Ilex microdonta & 3.7 & 0.04 & 0.35 & 0.02 & 0.19 & 41.0 & 41.0 \\
\hline 48 Orthosia dusenii & 3.7 & 0.04 & 0.35 & 0.02 & 0.19 & 62.0 & 62.0 \\
\hline 49 NI 5 & 3.7 & 0.04 & 0.35 & 0.02 & 0.19 & 30.0 & 30.0 \\
\hline 50 Verbesina glabrata & 3.7 & 0.04 & 0.35 & 0.02 & 0.19 & 40.0 & 40.0 \\
\hline Total & 1044.4 & 149.33 & 100 & 100 & 100 & & \\
\hline
\end{tabular}

Table 8. Phytosociological parameters of the grassy physiognomy of the upper montane grasslands in Serra da Igreja, $\mathrm{n}=14$. FA: absolute frequency (\%); Co: absolute cover (\%); FR: relative frequency (\%); CoR: relative cover (\%); PI: importance percentual (\%); Hpre: predominant height $(\mathrm{cm})$; Hmax: maximum height $(\mathrm{cm})$.

\begin{tabular}{|c|c|c|c|c|c|c|c|c|}
\hline & Species & FA & Co & FR & $\mathrm{CoR}$ & PI & Hpre & Hmax \\
\hline 1 & Cryptangium triquetrum & 100.0 & 62.14 & 10.07 & 41.29 & 25.68 & 64.4 & 75.2 \\
\hline 2 & Croton mullerianus & 100.0 & 39.29 & 10.07 & 26.10 & 18.09 & 50.4 & 61.1 \\
\hline 3 & Tibouchina dubia & 71.4 & 8.00 & 7.19 & 5.32 & 6.25 & 46.2 & 52.6 \\
\hline 4 & Myrsine altomontana & 85.7 & 3.43 & 8.63 & 2.28 & 5.46 & 50.2 & 54.9 \\
\hline 5 & Alstroemeria amabilis & 78.6 & 2.79 & 7.91 & 1.85 & 4.88 & 52.4 & 58.2 \\
\hline 6 & Handroanthus catarinesis & 50.0 & 5.29 & 5.04 & 3.51 & 4.27 & 57.9 & 61.4 \\
\hline 7 & Xyris stenophylla & 42.9 & 4.79 & 4.32 & 3.18 & 3.75 & 53.3 & 64.2 \\
\hline 8 & Galium hypocarpium ssp. indecorum & 50.0 & 1.79 & 5.04 & 1.19 & 3.11 & 38.3 & 40.3 \\
\hline 9 & Sticherus sp. & 35.7 & 3.21 & 3.60 & 2.14 & 2.87 & 55.6 & 58.0 \\
\hline 10 & Leandra cordigera & 35.7 & 2.14 & 3.60 & 1.42 & 2.51 & 48.2 & 50.6 \\
\hline 11 & Utricularia reniformis & 35.7 & 0.86 & 3.60 & 0.57 & 2.08 & 17.8 & 19.6 \\
\hline 12 & Sticherus pruinosus & 28.6 & 1.14 & 2.88 & 0.76 & 1.82 & 35.3 & 42.0 \\
\hline 13 & Blechnum sp. 1 & 28.6 & 0.71 & 2.88 & 0.47 & 1.68 & 47.3 & 48.3 \\
\hline 14 & Siphoneugena reitzii & 28.6 & 0.57 & 2.88 & 0.38 & 1.63 & 56.8 & 58.0 \\
\hline 15 & Gaylussacia brasiliensis var. brasiliensis & 28.6 & 0.36 & 2.88 & 0.24 & 1.56 & 26.0 & 26.5 \\
\hline 16 & Eriocaulon ligulatum & 7.1 & 3.57 & 0.72 & 2.37 & 1.55 & 22.0 & 30.0 \\
\hline 17 & Dichanthelium sabulorum & 21.4 & 1.36 & 2.16 & 0.90 & 1.53 & 43.7 & 49.7 \\
\hline 18 & Symplocos corymboclados & 21.4 & 0.43 & 2.16 & 0.28 & 1.22 & 57.7 & 59.7 \\
\hline 19 & Hesperozygis rhododon & 14.3 & 1.43 & 1.44 & 0.95 & 1.19 & 47.5 & 57.5 \\
\hline 20 & Vernonanthura montevidensis & 14.3 & 1.29 & 1.44 & 0.85 & 1.15 & 64.5 & 78.5 \\
\hline 21 & Weinmannia humilis & 14.3 & 0.71 & 1.44 & 0.47 & 0.96 & 61.5 & 61.5 \\
\hline 22 & Agarista niederleinii var. niederleinii & 14.3 & 0.57 & 1.44 & 0.38 & 0.91 & 73.0 & 78.5 \\
\hline & Poaceae 2 & 7.1 & 1.43 & 0.72 & 0.95 & 0.83 & 59.0 & 75.0 \\
\hline & Baccharis angusticeps & 14.3 & 0.21 & 1.44 & 0.14 & 0.79 & 23.5 & 23.5 \\
\hline & Verbesina glabrata & 7.1 & 0.86 & 0.72 & 0.57 & 0.64 & 60.0 & 62.0 \\
\hline & Myrtaceae indet. 1 & 7.1 & 0.71 & 0.72 & 0.47 & 0.60 & 15.0 & 27.0 \\
\hline
\end{tabular}


Table 8 (continuation)

\begin{tabular}{llccccccc}
\hline Species & FA & Co & FR & CoR & PI & Hpre & Hmax \\
\hline 27 & Myrcia pulchra & 7.1 & 0.50 & 0.72 & 0.33 & 0.53 & 76.0 & 79.0 \\
28 & Chusquea mimosa & 7.1 & 0.29 & 0.72 & 0.19 & 0.45 & 215.0 & 215.0 \\
29 & Graphistylis serrana & 7.1 & 0.21 & 0.72 & 0.14 & 0.43 & 39.0 & 54.0 \\
30 & Baccharis crispa & 7.1 & 0.14 & 0.72 & 0.09 & 0.41 & 49.0 & 49.0 \\
31 & Ilex microdonta & 7.1 & 0.14 & 0.72 & 0.09 & 0.41 & 56.0 & 56.0 \\
32 & Danthonia sp. 1 & 7.1 & 0.07 & 0.72 & 0.05 & 0.38 & 41.0 & 41.0 \\
33 & Ocotea porosa & 7.1 & 0.07 & 0.72 & 0.05 & 0.38 & 38.0 & 38.0 \\
\hline Total & 992.9 & 150.50 & 100 & 100 & 100 & & \\
\hline
\end{tabular}

Table 9. Phytosociological parameters of the shrubby physiognomy of the upper montane grasslands in Serra da Prata, $\mathrm{n}=15$. FA: absolute frequency (\%); Co: absolute cover (\%); FR: relative frequency (\%); CoR: relative cover (\%); PI: importance percentual (\%); Hpre: predominant height (cm); Hmax: maximum height $(\mathrm{cm})$.

\begin{tabular}{lrrrrrrr}
\hline Species & FA & Co & FR & CoR & PI & Hpre & Hmax \\
\hline 1 Croton mullerianus & 100.0 & 36.60 & 9.37 & 30.23 & 19.80 & 53.9 & 62.3 \\
2 Cryptangium triquetrum & 93.3 & 13.87 & 8.75 & 11.45 & 10.10 & 56.6 & 68.9 \\
3 Machaerina austrobrasiliensis & 80.0 & 13.87 & 7.50 & 11.45 & 9.48 & 61.8 & 81.8 \\
4 Tibouchina dubia & 80.0 & 13.40 & 7.50 & 11.07 & 9.28 & 59.5 & 61.9 \\
5 Sticherus sp. & 66.7 & 7.53 & 6.25 & 6.22 & 6.24 & 44.7 & 52.0 \\
6 Chusquea mimosa & 66.7 & 5.60 & 6.25 & 4.63 & 5.44 & 68.9 & 96.5 \\
7 Poaceae 1 & 53.3 & 2.87 & 5.00 & 2.37 & 3.68 & 42.3 & 53.0 \\
8 Myrcia pulchra & 40.0 & 4.27 & 3.75 & 3.52 & 3.64 & 70.3 & 79.7 \\
9 Gaylussacia brasiliensis var. brasiliensis & 53.3 & 1.67 & 5.00 & 1.38 & 3.19 & 38.3 & 40.6 \\
10 Myrsine altomontana & 46.7 & 2.20 & 4.38 & 1.82 & 3.10 & 63.7 & 68.0 \\
11 Gaultheria serrata var. organensis & 53.3 & 0.93 & 5.00 & 0.77 & 2.89 & 46.9 & 48.1 \\
12 Vriesea platynema var. variegata & 20.0 & 3.80 & 1.88 & 3.14 & 2.51 & 57.0 & 68.3 \\
13 Alstroemeria amabilis & 40.0 & 1.40 & 3.75 & 1.16 & 2.45 & 38.2 & 42.7 \\
14 Xyris piraquarae & 26.7 & 2.47 & 2.50 & 2.04 & 2.27 & 54.5 & 62.5 \\
15 Hesperozygis rhododon & 26.7 & 1.80 & 2.50 & 1.49 & 1.99 & 69.0 & 83.8 \\
16 Chusquea pinifolia & 20.0 & 1.73 & 1.88 & 1.43 & 1.65 & 58.3 & 75.0 \\
17 Vernonanthura montevidensis & 20.0 & 0.73 & 1.88 & 0.61 & 1.24 & 69.3 & 72.3 \\
18 Utricularia reniformis & 20.0 & 0.40 & 1.88 & 0.33 & 1.10 & 24.7 & 24.7 \\
19 Graphistylis serrana & 13.3 & 0.80 & 1.25 & 0.66 & 0.96 & 41.0 & 47.0 \\
20 Leandra quinquedentata & 13.3 & 0.73 & 1.25 & 0.61 & 0.93 & 56.0 & 61.5 \\
21 Blechnum cordatum & 13.3 & 0.47 & 1.25 & 0.39 & 0.82 & 41.0 & 44.0 \\
22 Leandra cordigera & 13.3 & 0.47 & 1.25 & 0.39 & 0.82 & 41.5 & 44.5 \\
23 Smilax campestris & 13.3 & 0.47 & 1.25 & 0.39 & 0.82 & 69.5 & 75.0 \\
24 Galium hypocarpium ssp. indecorum & 13.3 & 0.33 & 1.25 & 0.28 & 0.76 & 57.0 & 60.0 \\
25 Orthosia dusenii & 6.7 & 1.07 & 0.63 & 0.88 & 0.75 & 80.0 & 102.0 \\
26 Ternstroemia brasiliensis & 6.7 & 0.33 & 0.63 & 0.28 & 0.45 & 58.0 & 63.0 \\
27 Siphoneugena reitzii & 6.7 & 0.27 & 0.63 & 0.22 & 0.42 & 39.0 & 46.0 \\
28 Baccharis angusticeps & 6.7 & 0.20 & 0.63 & 0.17 & 0.40 & 52.0 & 52.0 \\
29 Baccharis crispa & 6.7 & 0.20 & 0.63 & 0.17 & 0.40 & 67.0 & 76.0
\end{tabular}


Table 9 (continuation)

\begin{tabular}{lrrrrrrr}
\hline Species & FA & Co & FR & CoR & PI & Hpre & Hmax \\
\hline 30 Coccocypselum sp. & 6.7 & 0.13 & 0.63 & 0.11 & 0.37 & 47.0 & 47.0 \\
31 Handroanthus catarinesis & 6.7 & 0.13 & 0.63 & 0.11 & 0.37 & 111.0 & 111.0 \\
32 Dendrophorbium limosum & 6.7 & 0.07 & 0.63 & 0.06 & 0.34 & 69.0 & 69.0 \\
33 Doryopteris crenulans & 6.7 & 0.07 & 0.63 & 0.06 & 0.34 & 15.0 & 15.0 \\
34 Oxypetalum sp. & 6.7 & 0.07 & 0.63 & 0.06 & 0.34 & 38.0 & 38.0 \\
35 Rhynchospora sp. 4 & 6.7 & 0.07 & 0.63 & 0.06 & 0.34 & 58.0 & 58.0 \\
36 Symplocos corymboclados & 6.7 & 0.07 & 0.63 & 0.06 & 0.34 & 31.0 & 33.0 \\
\hline Total & 1066.7 & 121.07 & 100 & 100 & 100 & & \\
\hline
\end{tabular}

Table 10. Phytosociological parameters of the grassy physiognomy of the upper montane grasslands in Serra da Prata, $\mathrm{n}=15$. FA: absolute frequency (\%); Co: absolute cover (\%); FR: relative frequency (\%); CoR: relative cover (\%); PI: importance percentual (\%); Hpre: predominant height $(\mathrm{cm})$; Hmax: maximum height $(\mathrm{cm})$.

\begin{tabular}{|c|c|c|c|c|c|c|c|c|}
\hline & Species & FA & $\mathrm{Co}$ & FR & CoR & PI & Hpre & Hmax \\
\hline 1 & Cryptangium triquetrum & 85.7 & 26.71 & 7.89 & 21.42 & 14.66 & 52.5 & 74.8 \\
\hline 2 & Croton mullerianus & 100.0 & 20.14 & 9.21 & 16.15 & 12.68 & 50.0 & 57.6 \\
\hline 3 & Machaerina austrobrasiliensis & 85.7 & 13.86 & 7.89 & 11.11 & 9.50 & 64.0 & 80.8 \\
\hline 4 & Gaylussacia brasiliensis var brasiliensis & 85.7 & 8.71 & 7.89 & 6.99 & 7.44 & 33.3 & 36.8 \\
\hline 5 & Xyris stenophylla & 57.1 & 10.71 & 5.26 & 8.59 & 6.93 & 24.8 & 32.3 \\
\hline 6 & Xyris piraquarae & 42.9 & 12.29 & 3.95 & 9.85 & 6.90 & 52.7 & 77.7 \\
\hline 7 & Sticherus sp. & 85.7 & 4.57 & 7.89 & 3.67 & 5.78 & 36.8 & 41.2 \\
\hline 8 & Tibouchina dubia & 71.4 & 5.71 & 6.58 & 4.58 & 5.58 & 40.4 & 44.2 \\
\hline 9 & Chusquea mimosa & 42.9 & 2.86 & 3.95 & 2.29 & 3.12 & 62.3 & 70.3 \\
\hline 11 & Poaceae 1 & 42.9 & 0.86 & 3.95 & 0.69 & 2.32 & 52.7 & 56.7 \\
\hline 10 & Phalocallis geniculata & 42.9 & 0.86 & 3.95 & 0.69 & 2.32 & 45.7 & 50.7 \\
\hline 12 & Vriesea platynema var. variegata & 14.3 & 4.00 & 1.32 & 3.21 & 2.26 & 59.0 & 72.0 \\
\hline 13 & Sticherus pruinosus & 28.6 & 1.43 & 2.63 & 1.15 & 1.89 & 60.0 & 68.5 \\
\hline 14 & Myrcia hartwegiana & 28.6 & 1.29 & 2.63 & 1.03 & 1.83 & 85.5 & 239.0 \\
\hline 15 & Chusquea pinifolia & 28.6 & 1.14 & 2.63 & 0.92 & 1.77 & 52.0 & 57.0 \\
\hline 16 & Alstroemeria amabilis & 28.6 & 0.57 & 2.63 & 0.46 & 1.54 & 60.5 & 61.5 \\
\hline 17 & Clethra uleana & 14.3 & 2.14 & 1.32 & 1.72 & 1.52 & 95.0 & 102.0 \\
\hline 18 & Myrsine altomontana & 28.6 & 0.43 & 2.63 & 0.34 & 1.49 & 61.0 & 67.0 \\
\hline 19 & Hesperozygis rhododon & 14.3 & 1.57 & 1.32 & 1.26 & 1.29 & 84.0 & 92.0 \\
\hline 20 & Symplocos corymboclados & 14.3 & 1.29 & 1.32 & 1.03 & 1.17 & 60.0 & 66.0 \\
\hline 21 & Eriocaulon ligulatum & 14.3 & 1.14 & 1.32 & 0.92 & 1.12 & 24.0 & 43.0 \\
\hline 23 & Myrcia pulchra & 14.3 & 0.43 & 1.32 & 0.34 & 0.83 & 60.0 & 64.0 \\
\hline 22 & Gaultheria serrata var. organensis & 14.3 & 0.43 & 1.32 & 0.34 & 0.83 & 53.0 & 67.0 \\
\hline 25 & Siphoneugena reitzii & 14.3 & 0.29 & 1.32 & 0.23 & 0.77 & 95.0 & 95.0 \\
\hline 24 & Galium hypocarpium ssp. indecorum & 14.3 & 0.29 & 1.32 & 0.23 & 0.77 & 64.0 & 64.0 \\
\hline 26 & Tibouchina hospita & 14.3 & 0.29 & 1.32 & 0.23 & 0.77 & 35.0 & 35.0 \\
\hline 27 & Utricularia reniformis & 14.3 & 0.29 & 1.32 & 0.23 & 0.77 & 25.0 & 25.0 \\
\hline 28 & Baccharis angusticeps & 14.3 & 0.14 & 1.32 & 0.11 & 0.72 & 55.0 & 55.0 \\
\hline 29 & Dendrophorbium limosum & 14.3 & 0.14 & 1.32 & 0.11 & 0.72 & 35.0 & 35.0 \\
\hline \multirow[t]{2}{*}{30} & Leandra cordigera & 14.3 & 0.14 & 1.32 & 0.11 & 0.72 & 17.0 & 17.0 \\
\hline & Total & 1085.7 & 124.71 & 100 & 100 & 100 & & \\
\hline
\end{tabular}


Table 11. Phytosociological parameters of the shrubby physiognomy of the upper montane grasslands in Serra da Farinha Seca, $\mathrm{n}=10$. FA: absolute frequency (\%); Co: absolute cover (\%); FR: relative frequency (\%); CoR: relative cover (\%); PI: importance percentual (\%); Hpre: predominant height (cm); Hmax: maximum height (cm).

\begin{tabular}{|c|c|c|c|c|c|c|c|c|}
\hline & Species & FA & $\mathrm{Co}$ & FR & CoR & PI & Hpre & Hmax \\
\hline 1 & Mimosa congestifolia & 80 & 32.50 & 6.78 & 24.13 & 15.45 & 69.0 & 80.6 \\
\hline 2 & Cryptangium triquetrum & 80 & 13.20 & 6.78 & 9.80 & 8.29 & 53.8 & 57.8 \\
\hline 3 & Poaceae 09 & 70 & 10.20 & 5.93 & 7.57 & 6.75 & 58.0 & 79.1 \\
\hline 4 & Sticherus sp. & 70 & 9.60 & 5.93 & 7.13 & 6.53 & 41.1 & 48.4 \\
\hline 5 & Chusquea pinifolia & 70 & 8.80 & 5.93 & 6.53 & 6.23 & 79.0 & 85.1 \\
\hline 6 & Siphoneugena reitzii & 60 & 9.00 & 5.08 & 6.68 & 5.88 & 65.5 & 70.5 \\
\hline 7 & Myrcia hartwegiana & 40 & 9.70 & 3.39 & 7.20 & 5.30 & 65.3 & 83.8 \\
\hline 8 & Machaerina austrobrasiliensis & 70 & 5.10 & 5.93 & 3.79 & 4.86 & 59.4 & 77.0 \\
\hline 9 & Leandra cordigera & 70 & 3.70 & 5.93 & 2.75 & 4.34 & 29.9 & 40.4 \\
\hline 10 & Ilex microdonta & 30 & 5.50 & 2.54 & 4.08 & 3.31 & 57.0 & 68.0 \\
\hline 11 & Rhynchospora sp. 1 & 40 & 4.10 & 3.39 & 3.04 & 3.22 & 52.0 & 59.0 \\
\hline 12 & Myrsine altomontana & 50 & 1.50 & 4.24 & 1.11 & 2.68 & 52.4 & 47.4 \\
\hline 13 & Tibouchina dubia & 30 & 2.60 & 2.54 & 1.93 & 2.24 & 34.0 & 35.3 \\
\hline 14 & Clethra uleana & 30 & 2.20 & 2.54 & 1.63 & 2.09 & 62.3 & 73.7 \\
\hline 15 & Sticherus pruinosus & 20 & 3.00 & 1.69 & 2.23 & 1.96 & 42.0 & 42.5 \\
\hline 16 & Gaylussacia brasiliensis var brasiliensis & 40 & 0.70 & 3.39 & 0.52 & 1.95 & 50.0 & 53.3 \\
\hline 17 & Galianthe gertii & 20 & 2.30 & 1.69 & 1.71 & 1.70 & 49.5 & 56.5 \\
\hline 18 & Psidium ovale & 20 & 1.30 & 1.69 & 0.97 & 1.33 & 58.0 & 77.5 \\
\hline 19 & Myrcia guianensis & 20 & 1.20 & 1.69 & 0.89 & 1.29 & 79.0 & 90.0 \\
\hline 20 & Galium hypocarpium ssp. indecorum & 20 & 1.00 & 1.69 & 0.74 & 1.22 & 53.0 & 56.0 \\
\hline 21 & Leandra quinquedentata & 20 & 0.80 & 1.69 & 0.59 & 1.14 & 55.5 & 52.5 \\
\hline 22 & Persea alba & 10 & 1.80 & 0.85 & 1.34 & 1.09 & 110.0 & 133.0 \\
\hline 23 & Poaceae 7 & 20 & 0.50 & 1.69 & 0.37 & 1.03 & 45.0 & 54.0 \\
\hline 24 & NI 3 & 20 & 0.40 & 1.69 & 0.30 & 1.00 & 35.5 & 36.5 \\
\hline 25 & Mikania clematidifolia & 10 & 0.60 & 0.85 & 0.45 & 0.65 & 43.0 & 44.0 \\
\hline 26 & Vriesea platynema var. variegata & 10 & 0.50 & 0.85 & 0.37 & 0.61 & 40.0 & 44.0 \\
\hline 27 & Graphistylis serrana & 10 & 0.40 & 0.85 & 0.30 & 0.57 & 53.0 & 44.0 \\
\hline 28 & Cyperaceae 3 & 10 & 0.30 & 0.85 & 0.22 & 0.54 & 65.0 & 65.0 \\
\hline 29 & Rubiaceae & 10 & 0.30 & 0.85 & 0.22 & 0.54 & 32.0 & 35.0 \\
\hline 30 & Vernonanthura montevidensis & 10 & 0.30 & 0.85 & 0.22 & 0.54 & 37.0 & 100.0 \\
\hline 31 & Alstroemeria amabilis & 10 & 0.20 & 0.85 & 0.15 & 0.50 & 40.0 & 40.0 \\
\hline 32 & Poaceae 6 & 10 & 0.20 & 0.85 & 0.15 & 0.50 & 32.0 & 32.0 \\
\hline 33 & Smilax campestris & 10 & 0.20 & 0.85 & 0.15 & 0.50 & 54.0 & 64.0 \\
\hline 34 & Xyris piraquarae & 10 & 0.20 & 0.85 & 0.15 & 0.50 & 66.0 & 77.0 \\
\hline 35 & Apocynaceae 1 & 10 & 0.10 & 0.85 & 0.07 & 0.46 & 44.0 & 44.0 \\
\hline 36 & Eryngium koehneanum & 10 & 0.10 & 0.85 & 0.07 & 0.46 & 40.0 & 40.0 \\
\hline 37 & Podocarpus sellowii & 10 & 0.10 & 0.85 & 0.07 & 0.46 & 45.0 & 47.0 \\
\hline 38 & Symplocos corymboclados & 10 & 0.10 & 0.85 & 0.07 & 0.46 & 46.0 & 46.0 \\
\hline 39 & Handroanthus catarinesis & 10 & 0.10 & 0.85 & 0.07 & 0.46 & 62.0 & 62.0 \\
\hline 40 & Tibouchina hospita & 10 & 0.10 & 0.85 & 0.07 & 0.46 & 39.0 & 39.0 \\
\hline 41 & Utricularia reniformis & 10 & 0.10 & 0.85 & 0.07 & 0.46 & 28.0 & 28.0 \\
\hline & Verbesina glabrata & 10 & 0.10 & 0.85 & 0.07 & 0.46 & 76.0 & 76.0 \\
\hline & Total & 1180 & 134.7 & 100 & 100 & 100 & & \\
\hline
\end{tabular}


ridges (approx. $800 \mathrm{~m}$ asl) in Serra do Mar State Park- Núcleo Curucutu (SP). In the sampling of Serra Gigante, Cryptangium triquetrum was found in all sampled plots, comprising $52 \%$ of the relative cover, which corresponds to almost one third of the importance percentage. Paspalum polyphyllum stood out mainly for its cover percentage as well as Tibouchina hatschbachii due to the species high frequency. The latter was detected exclusively in Serra Gigante, but showed importance among the community structure. Neomarica caerulea is another species found exclusively in Serra Gigante and showed great importance as well.

We observed that, in general, the plant community had lower heights, in which the average height of the five major species was lower than $50 \mathrm{~cm}$. Altogether, these species accounted for more than $80 \%$ of the community relative cover.

Structure of upper montane grasslands in Serra da Pedra Branca do Araraquara

Serra da P.B. do Araraquara - Physiognomy: shrubby - 31 species were found in the seven sampled plots within an absolute cover of $124.9 \mathrm{~m}^{2} \cdot 100^{-1} \mathrm{~m}^{2}$ (table 15). Sticherus sp. stood out as the most important species, especially for presenting high cover percentage, comprising $22 \%$ of the community. Tibouchina dubia, Vernonanthura montevidensis and Eryngium koehneanum, despite being the most frequent, presented lower vegetation cover when compared to Sticherus sp. Coccocypselum condalia and Valeriana ulei also showed high frequency, but much lower cover. Therefore, these five species were assigned to the lower strata of the community. Machaerina austrobrasiliensis stood out for its high cover values as well as for its predominant and maximum heights, being assigned to the upper strata.

Serra da P.B. do Araraquara - Physiognomy: grassy 22 species were found in the five sampled plots within an absolute cover of $107.4 \mathrm{~m}^{2} .100^{-1} \mathrm{~m}^{2}$ (table 16). Cryptangium triquetrum was the most important species, followed by Eryngium koehneanum, Tibouchina dubia and a non-identified Poaceae. These four species comprised more than $60 \%$ of the importance percentage and nearly $80 \%$ of the cover percentage.

Structure of upper montane grasslands in the State of Paraná, Southern Brazil

From the 280 vascular plant species surveyed by the same authors (Mocochinski \& Scheer, 2008), 195 species were sampled in at least one of the 324 plots of $1 \mathrm{~m}^{2}$, distributed in the upper montane grasslands of six mountain ranges from Southern Brazil, which were the aim of the present study.

Based upon our findings, Cryptangium triquetrum was the most important and frequent species in the upper montane grassland structures (approximately 14\%), since it was found in all grassland physiognomies within several mountain ranges studied (figure 2).

Croton mullerianus was the second most dominant species (12\%), especially in shrubby physiognomies, although it was not found neither in grassy and shrubby physiognomies of Serra da Farinha Seca and Serra da Pedra Branca do Araraquara, nor in Chusquea pinifolia physiognomy of Serra da Farinha Seca (figure 2).

Chusquea pinifolia was the third most important species $(7 \%)$, based on its remarkable occurrence in both Serra do Ibitiraquire and Serra da Prata, by prevailing in $C$. pinifolia physiognomy.

These three main species resulted in approximately $33 \%$ of importance of the general phytosociological survey carried out in the upper montane grasslands. As for the top 10 species, which also include Machaerina austrobrasiliensis, Deschampsia caespitosa, Gleichenella pectitata, Tibouchina dubia, Xyris stenophylla, Eryngium koehnearum and Eriochrysis holcoides accounted for $50 \%$ of the relative importance (figure 2).

It is noteworthy that out of these 10 most important species in the upper montane grasslands in the State of Paraná, only three were cited in the List of Species related to Altitude Grasslands (campos de altitude, that include or at least should include species from montane and upper montane grasslands) of Southern Brazil by CONAMA Resolution 423/2010: Machaerina austrobrasiliensis, Deschampsia caespitosa and Eriochrysis holcoides are classified as indicator species of primary (mature/old growth) vegetation formations as well as medium or advanced stages of regeneration. The first one is also classified as endemic or rare. The other two species were also included in the List of Northeastern Brazil of the same Resolution, however, they should also be included in the List of the Southeastern Region. As a matter of fact, Chusquea pinifolia, the third main species of the present study, has not been cited in the List of the Southern Region, although it has been included in the List of both Northeastern and Southeastern Regions. Croton mullerianus (second main species in this survey) is not 
Table 12. Phytosociological parameters of the grassy physiognomy of the upper montane grasslands in Serra da Farinha Seca, $n=22$. FA: absolute frequency ( $\%)$; Co: absolute cover $(\%)$; FR: relative frequency $(\%)$; CoR: relative cover $(\%)$; PI: importance percentual (\%); Hpre: predominant height (cm); Hmax: maximum height (cm).

\begin{tabular}{|c|c|c|c|c|c|c|c|c|}
\hline & Species & FA & $\mathrm{Co}$ & FR & CoR & PI & Hpre & Hmax \\
\hline 1 & Cryptangium triquetrum & 100.0 & 72.50 & 8.09 & 49.05 & 28.57 & 62.9 & 79.1 \\
\hline 2 & Siphoneugena reitzii & 81.8 & 6.05 & 6.62 & 4.09 & 5.35 & 57.3 & 63.6 \\
\hline 3 & Tibouchina dubia & 72.7 & 6.05 & 5.88 & 4.09 & 4.99 & 44.6 & 50.8 \\
\hline 4 & Eryngium koehneanum & 63.6 & 4.95 & 5.15 & 3.35 & 4.25 & 44.1 & 50.9 \\
\hline 5 & Baccharis nebularis & 68.2 & 4.09 & 5.51 & 2.77 & 4.14 & 53.1 & 60.2 \\
\hline 6 & Galianthe gertii & 45.5 & 4.32 & 3.68 & 2.92 & 3.30 & 34.2 & 38.3 \\
\hline 7 & Poaceae 6 & 54.5 & 2.45 & 4.41 & 1.66 & 3.04 & 33.6 & 45.0 \\
\hline 8 & Sticherus pruinosus & 50.0 & 2.91 & 4.04 & 1.97 & 3.01 & 45.9 & 49.6 \\
\hline 9 & Sticherus sp. & 27.3 & 5.32 & 2.21 & 3.60 & 2.90 & 51.5 & 55.5 \\
\hline 10 & Myrcia hartwegiana & 31.8 & 4.32 & 2.57 & 2.92 & 2.75 & 65.1 & 72.7 \\
\hline 11 & Xyris dissitifolia & 50.0 & 2.05 & 4.04 & 1.38 & 2.71 & 43.6 & 47.1 \\
\hline 12 & Vriesea platynema var. variegata & 22.7 & 4.59 & 1.84 & 3.11 & 2.47 & 39.4 & 53.6 \\
\hline 13 & Utricularia reniformis & 50.0 & 0.91 & 4.04 & 0.62 & 2.33 & 31.9 & 35.4 \\
\hline 14 & Myrsine altomontana & 45.5 & 1.23 & 3.68 & 0.83 & 2.25 & 48.1 & 54.4 \\
\hline 15 & Leandra quinquedentata & 36.4 & 2.09 & 2.94 & 1.41 & 2.18 & 51.9 & 56.6 \\
\hline 16 & Graphistylis serrana & 27.3 & 2.18 & 2.21 & 1.48 & 1.84 & 44.5 & 49.3 \\
\hline 17 & Clethra uleana & 18.2 & 2.68 & 1.47 & 1.81 & 1.64 & 72.5 & 82.3 \\
\hline 18 & Galium hypocarpium ssp. indecorum & 31.8 & 0.95 & 2.57 & 0.65 & 1.61 & 37.9 & 44.4 \\
\hline 19 & Poaceae 9 & 18.2 & 2.45 & 1.47 & 1.66 & 1.57 & 44.0 & 61.5 \\
\hline 20 & Weinmannia humilis & 13.6 & 2.45 & 1.10 & 1.66 & 1.38 & 62.3 & 74.3 \\
\hline 21 & Leandra cordigera & 22.7 & 1.36 & 1.84 & 0.92 & 1.38 & 49.2 & 51.8 \\
\hline 22 & Poaceae 7 & 22.7 & 0.91 & 1.84 & 0.62 & 1.23 & 37.8 & 52.4 \\
\hline 23 & Mimosa congestifolia & 22.7 & 0.73 & 1.84 & 0.49 & 1.17 & 67.4 & 72.4 \\
\hline 24 & Baccharis crispa & 18.2 & 1.18 & 1.47 & 0.80 & 1.14 & 45.8 & 51.5 \\
\hline 25 & Chusquea pinifolia & 9.1 & 1.45 & 0.74 & 0.98 & 0.86 & 64.5 & 72.5 \\
\hline 26 & Handroanthus catarinesis & 13.6 & 0.82 & 1.10 & 0.55 & 0.83 & 52.3 & 54.3 \\
\hline 27 & Tibouchina hospita & 18.2 & 0.27 & 1.47 & 0.18 & 0.83 & 27.8 & 28.5 \\
\hline 28 & Gaylussacia brasiliensis var brasiliensis & 18.2 & 0.18 & 1.47 & 0.12 & 0.80 & 31.8 & 32.0 \\
\hline 29 & Machaerina austrobrasiliensis & 9.1 & 1.00 & 0.74 & 0.68 & 0.71 & 54.0 & 70.0 \\
\hline 30 & Psidium ovale & 9.1 & 0.82 & 0.74 & 0.55 & 0.64 & 68.5 & 96.0 \\
\hline 31 & Baccharis angusticeps & 13.6 & 0.27 & 1.10 & 0.18 & 0.64 & 52.0 & 56.7 \\
\hline 32 & Lycopodium clavatum & 13.6 & 0.23 & 1.10 & 0.15 & 0.63 & 34.0 & 37.7 \\
\hline 33 & Symplocos corymboclados & 9.1 & 0.68 & 0.74 & 0.46 & 0.60 & 71.5 & 74.0 \\
\hline 34 & Xyris piraquarae & 9.1 & 0.59 & 0.74 & 0.40 & 0.57 & 69.0 & 81.5 \\
\hline 35 & Coccocypselum sp. & 9.1 & 0.45 & 0.74 & 0.31 & 0.52 & 33.0 & 34.5 \\
\hline 36 & Apocynaceae 1 & 9.1 & 0.18 & 0.74 & 0.12 & 0.43 & 45.5 & 49.0 \\
\hline 37 & Mikania clematidifolia & 9.1 & 0.18 & 0.74 & 0.12 & 0.43 & 48.0 & 51.0 \\
\hline 38 & Alstroemeria amabilis & 9.1 & 0.14 & 0.74 & 0.09 & 0.41 & 43.5 & 46.0 \\
\hline 39 & Poaceae 8 & 9.1 & 0.14 & 0.74 & 0.09 & 0.41 & 38.5 & 42.0 \\
\hline 40 & Gaultheria serrata var. organensis & 9.1 & 0.09 & 0.74 & 0.06 & 0.40 & 28.5 & 30.0 \\
\hline 41 & Mikania paranensis & 9.1 & 0.09 & 0.74 & 0.06 & 0.40 & 49.0 & 50.0 \\
\hline
\end{tabular}


Table 12 (continuation)

\begin{tabular}{llccccccc}
\hline Species & FA & Co & FR & CoR & PI & Hpre & Hmax \\
\hline 42 & Myrtaceae indet. & 4.5 & 0.45 & 0.37 & 0.31 & 0.34 & 62.0 & 68.0 \\
43 & Ocotea tristis & 4.5 & 0.36 & 0.37 & 0.25 & 0.31 & 77.0 & 83.0 \\
44 & Austroeupatorium neglectum & 4.5 & 0.09 & 0.37 & 0.06 & 0.21 & 54.0 & 58.0 \\
45 & Dendrophorbium limosum & 4.5 & 0.09 & 0.37 & 0.06 & 0.21 & 48.0 & 51.0 \\
46 & Eugenia neomyrtifolia & 4.5 & 0.09 & 0.37 & 0.06 & 0.21 & 48.0 & 40.0 \\
47 & Ilex microdonta & 4.5 & 0.09 & 0.37 & 0.06 & 0.21 & 46.0 & 49.0 \\
48 & NI 3 & 4.5 & 0.09 & 0.37 & 0.06 & 0.21 & 44.0 & 54.0 \\
49 & Phalocallis geniculata & 4.5 & 0.05 & 0.37 & 0.03 & 0.20 & 36.0 & 36.0 \\
50 & Podocarpus sellowii & 4.5 & 0.05 & 0.37 & 0.03 & 0.20 & 26.0 & 26.0 \\
51 & Smilax campestris & 4.5 & 0.05 & 0.37 & 0.03 & 0.20 & 35.0 & 39.0 \\
52 & Holocheilus brasiliensis & 4.5 & 0.05 & 0.37 & 0.03 & 0.20 & 30.0 & 33.0 \\
53 & Valeriana ulei & 4.5 & 0.05 & 0.37 & 0.03 & 0.20 & 62.0 & 62.0 \\
\hline & Total & 1236.4 & 147.82 & 100 & 100 & 100 & & \\
\hline
\end{tabular}

Table 13. Phytosociological parameters of the Chusquea pinifolia physiognomy of the upper montane grasslands in Serra da Farinha Seca, $\mathrm{n}=5$. FA: absolute frequency (\%); Co: absolute cover (\%); FR: relative frequency (\%); CoR: relative cover (\%); PI: importance percentual (\%); Hpre: predominant height (cm); Hmax: maximum height $(\mathrm{cm})$.

\begin{tabular}{llrrrrrrc}
\hline & Species & FA & Co & FR & \multicolumn{1}{c}{ CoR } & PI & Hpre & Hmax \\
\hline 1 & Chusquea pinifolia & 100 & 70.80 & 8.77 & 52.60 & 30.69 & 74.2 & 92.0 \\
2 & Mimosa congestifolia & 100 & 13.80 & 8.77 & 10.25 & 9.51 & 70.6 & 76.2 \\
3 & Siphoneugena reitzii & 100 & 7.60 & 8.77 & 5.65 & 7.21 & 62.4 & 70.6 \\
4 & Sticherus sp. & 80 & 6.40 & 7.02 & 4.75 & 5.89 & 41.5 & 47.0 \\
5 & Baccharis nebularis & 40 & 6.60 & 3.51 & 4.90 & 4.21 & 57.5 & 63.0 \\
6 & Machaerina austrobrasiliensis & 60 & 3.60 & 5.26 & 2.67 & 3.97 & 55.3 & 67.7 \\
7 & Symplocos corymboclados & 60 & 1.80 & 5.26 & 1.34 & 3.30 & 65.3 & 67.7 \\
8 & Utricularia reniformis & 60 & 1.00 & 5.26 & 0.74 & 3.00 & 12.3 & 12.3 \\
9 & Clethra scabra & 20 & 5.40 & 1.75 & 4.01 & 2.88 & 85.0 & 93.0 \\
10 & Poaceae 8 & 60 & 0.60 & 5.26 & 0.45 & 2.85 & 20.0 & 20.0 \\
11 & Dendrophorbium limosum & 40 & 1.40 & 3.51 & 1.04 & 2.27 & 42.0 & 47.5 \\
12 & Alstroemeria amabilis & 40 & 1.20 & 3.51 & 0.89 & 2.20 & 37.5 & 39.5 \\
13 & Smilax campestris & 40 & 0.80 & 3.51 & 0.59 & 2.05 & 42.5 & 44.5 \\
14 & Gaultheria serrata var. organensis & 40 & 0.60 & 3.51 & 0.45 & 1.98 & 44.0 & 47.5 \\
15 & Handroanthus catarinesis & 20 & 2.60 & 1.75 & 1.93 & 1.84 & 64.0 & 72.0 \\
16 & Myrsine altomontana & 20 & 1.60 & 1.75 & 1.19 & 1.47 & 59.0 & 70.0 \\
17 & Poaceae 9 & 20 & 1.60 & 1.75 & 1.19 & 1.47 & 75.0 & 98.0 \\
18 & Galium hypocarpium ssp. indecorum & 20 & 1.20 & 1.75 & 0.89 & 1.32 & 37.0 & 45.0 \\
19 & Tibouchina dubia & 20 & 1.20 & 1.75 & 0.89 & 1.32 & 52.0 & 57.0 \\
20 & Coccocypselum sp. & 20 & 1.00 & 1.75 & 0.74 & 1.25 & 5.0 & 10.0 \\
21 & Rubiaceae & 20 & 1.00 & 1.75 & 0.74 & 1.25 & 40.0 & 45.0 \\
22 & Cryptangium triquetrum & 20 & 0.60 & 1.75 & 0.45 & 1.10 & 61.0 & 61.0 \\
23 & Leandra quinquedentata & 20 & 0.60 & 1.75 & 0.45 & 1.10 & 22.0 & 28.0 \\
24 & Baccharis crispa & 20 & 0.40 & 1.75 & 0.30 & 1.03 & 39.0 & 42.0
\end{tabular}


Table 13 (continuation)

\begin{tabular}{llrcccccc}
\hline & Species & FA & Co & FR & CoR & PI & Hpre & Hmax \\
\hline 25 & Ilex microdonta & 20 & 0.40 & 1.75 & 0.30 & 1.03 & 72.0 & 76.0 \\
26 & Coccocypselum condalia & 20 & 0.20 & 1.75 & 0.15 & 0.95 & 18.0 & 18.0 \\
27 & Myrcia hartwegiana & 20 & 0.20 & 1.75 & 0.15 & 0.95 & 50.0 & 53.0 \\
28 & Leandra cordigera & 20 & 0.20 & 1.75 & 0.15 & 0.95 & 42.0 & 44.0 \\
29 & Ocotea tristis & 20 & 0.20 & 1.75 & 0.15 & 0.95 & 59.0 & 59.0 \\
\hline & Total & 1140 & 134.60 & 100 & 100 & 100 & & \\
\hline
\end{tabular}

Table 14. Phytosociological parameters of the grassy physiognomy of the upper montane grasslands in Serra Gigante, $\mathrm{n}=12$. FA: absolute frequency (\%); Co: absolute cover (\%); FR: relative frequency (\%); CoR: relative cover (\%); PI: importance percentual (\%); Hpre: predominant height $(\mathrm{cm})$; Hmax: maximum height $(\mathrm{cm})$.

\begin{tabular}{llrrrrrrc}
\hline & Species & FA & \multicolumn{1}{c}{ Co } & \multicolumn{1}{c}{ FR } & CoR & PI & Hpre & Hmax \\
\hline 1 & Cryptangium triquetrum & 100.0 & 59.00 & 12.77 & 52.48 & 32.62 & 43.4 & 53.5 \\
2 & Paspalum polyphyllum & 83.3 & 13.25 & 10.64 & 11.79 & 11.21 & 31.0 & 40.1 \\
3 & Tibouchina hatschbachii & 91.7 & 8.17 & 11.70 & 7.26 & 9.48 & 38.6 & 49.8 \\
4 & Neomarica caerulea & 75.0 & 3.83 & 9.57 & 3.41 & 6.49 & 41.6 & 47.8 \\
5 & Myrcia hartwegiana & 50.0 & 6.42 & 6.38 & 5.71 & 6.05 & 44.7 & 52.2 \\
6 & Siphoneugena reitzii & 58.3 & 4.00 & 7.45 & 3.56 & 5.50 & 67.6 & 78.4 \\
7 & Rhynchospora exaltata & 58.3 & 3.75 & 7.45 & 3.34 & 5.39 & 31.3 & 41.0 \\
8 & Dicranopteris nervosa & 50.0 & 3.75 & 6.38 & 3.34 & 4.86 & 25.0 & 30.0 \\
9 & Asteraceae 1 & 33.3 & 3.75 & 4.26 & 3.34 & 3.80 & 26.0 & 29.3 \\
10 & Poaceae 4 & 41.7 & 1.00 & 5.32 & 0.89 & 3.10 & 26.8 & 29.4 \\
11 & Mikania lanuginosa & 25.0 & 0.58 & 3.19 & 0.52 & 1.86 & 19.0 & 29.3 \\
12 & Gaylussacia brasiliensis var brasiliensis & 16.7 & 1.08 & 2.13 & 0.96 & 1.55 & 30.0 & 43.0 \\
13 & Pitcairnia flammea & 8.3 & 1.67 & 1.06 & 1.48 & 1.27 & 38.0 & 43.0 \\
14 & NI 1 & 16.7 & 0.17 & 2.13 & 0.15 & 1.14 & 25.0 & 25.0 \\
15 & Aechmea sp. & 8.3 & 0.50 & 1.06 & 0.44 & 0.75 & 32.0 & 32.0 \\
16 & Bulbostylis consanguinea & 8.3 & 0.50 & 1.06 & 0.44 & 0.75 & 11.0 & 11.0 \\
17 & Asteraceae 03 & 8.3 & 0.17 & 1.06 & 0.15 & 0.61 & 20.0 & 20.0 \\
18 & Coccocypselum condalia & 8.3 & 0.17 & 1.06 & 0.15 & 0.61 & 5.0 & 3.0 \\
19 & Mandevilla atroviolacea & 8.3 & 0.17 & 1.06 & 0.15 & 0.61 & 70.0 & 90.0 \\
20 & Piptocarpha densifolia & 8.3 & 0.17 & 1.06 & 0.15 & 0.61 & 34.0 & 34.0 \\
21 & Utricularia reniformis & 8.3 & 0.17 & 1.06 & 0.15 & 0.61 & 11.0 & 11.0 \\
22 & Pteridophyta 2 & 8.3 & 0.08 & 1.06 & 0.07 & 0.57 & 10.0 & 10.0 \\
23 & Ternstroemia brasiliensis & 8.3 & 0.08 & 1.06 & 0.07 & 0.57 & 56.0 & 56.0 \\
\hline & Total & 783.3 & 112.42 & 100 & 100 & 100 & & \\
\hline & & & & & & \\
& & & & & \\
& & & &
\end{tabular}

cited in the List. It is also noteworthy that Criptangium triquetum, structurally the most dominant species in this survey, is solely cited in the CONAMA Resolution to the Southeastern Region under the synonym Lagenocarpus triquetrus.

The four species with the highest percentage of importance on each physiognomy of each sampled subrange (table 17) showed, in most areas, approximately half the overall index for their respective communities.

In Serra do Ibitiraquire, Croton mullerianus stood out for being among the four main species in the five sampled physiognomies. Whereas, Cryptangium triquetrum was not only among the four main species in the physiognomy dominated by Machaerina 
Table 15. Phytosociological parameters of the shrubby physiognomy of the upper montane grasslands in Serra da Pedra Branca do Araraquara, $\mathrm{n}=7$. FA: absolute frequency; Co: absolute cover; FR: relative frequency (\%); CoR: relative cover (\%); PI: importance percentual (\%); Hpre: predominant height; Hmax: maximum height.

\begin{tabular}{|c|c|c|c|c|c|c|c|c|}
\hline & Species & FA & Co & FR & CoR & PI & Hpre & Hmax \\
\hline 1 & Sticherus sp. & 71.4 & 27.86 & 5.81 & 22.31 & 14.06 & 53.2 & 62.0 \\
\hline 2 & Tibouchina dubia & 85.7 & 14.29 & 6.98 & 11.44 & 9.21 & 54.5 & 66.7 \\
\hline 3 & Vernonanthura montevidensis & 85.7 & 11.86 & 6.98 & 9.50 & 8.24 & 74.0 & 88.0 \\
\hline 4 & Eryngium koehneanum & 85.7 & 9.43 & 6.98 & 7.55 & 7.26 & 62.2 & 70.3 \\
\hline 5 & Machaerina austrobrasiliensis & 57.1 & 11.57 & 4.65 & 9.27 & 6.96 & 90.3 & 108.3 \\
\hline 6 & Cryptangium triquetrum & 100.0 & 5.71 & 8.14 & 4.58 & 6.36 & 52.9 & 74.7 \\
\hline 7 & Siphoneugena reitzii & 57.1 & 8.86 & 4.65 & 7.09 & 5.87 & 73.0 & 87.5 \\
\hline 8 & Coccocypselum condalia & 85.7 & 2.86 & 6.98 & 2.29 & 4.63 & 23.3 & 30.7 \\
\hline 9 & Valeriana ulei & 71.4 & 1.14 & 5.81 & 0.92 & 3.36 & 37.8 & 44.3 \\
\hline 10 & Ericaceae 1 & 57.1 & 2.57 & 4.65 & 2.06 & 3.36 & 69.5 & 76.5 \\
\hline 11 & Xyris piraquarae & 42.9 & 3.86 & 3.49 & 3.09 & 3.29 & 51.3 & 61.7 \\
\hline 12 & Chusquea mimosa & 28.6 & 5.14 & 2.33 & 4.12 & 3.22 & 176.5 & 191.5 \\
\hline 13 & Holocheilus brasiliensis & 42.9 & 2.00 & 3.49 & 1.60 & 2.55 & 16.0 & 21.3 \\
\hline 14 & Leandra quinquedentata & 42.9 & 0.71 & 3.49 & 0.57 & 2.03 & 38.3 & 39.0 \\
\hline 15 & Myrtaceae 3 & 14.3 & 3.14 & 1.16 & 2.52 & 1.84 & 61.0 & 76.0 \\
\hline 16 & Myrsine altomontana & 28.6 & 1.43 & 2.33 & 1.14 & 1.73 & 73.0 & 87.0 \\
\hline 17 & Poaceae 12 & 14.3 & 2.86 & 1.16 & 2.29 & 1.73 & 44.0 & 55.0 \\
\hline 18 & Vriesea platynema var. variegata & 28.6 & 1.00 & 2.33 & 0.80 & 1.56 & 35.5 & 38.0 \\
\hline 19 & Miconia lymanii & 28.6 & 0.86 & 2.33 & 0.69 & 1.51 & 57.0 & 62.0 \\
\hline 20 & Bulbostylis consanguinea & 14.3 & 2.14 & 1.16 & 1.72 & 1.44 & 30.0 & 55.0 \\
\hline 21 & Agarista niederleinii var niederleinii & 28.6 & 0.29 & 2.33 & 0.23 & 1.28 & 43.0 & 43.0 \\
\hline 22 & Utricularia reniformis & 28.6 & 0.29 & 2.33 & 0.23 & 1.28 & 10.0 & 10.0 \\
\hline 23 & Blechnum cordatum & 14.3 & 1.57 & 1.16 & 1.26 & 1.21 & 49.0 & 52.0 \\
\hline 24 & Sticherus pruinosus & 14.3 & 1.43 & 1.16 & 1.14 & 1.15 & 45.0 & 51.0 \\
\hline 25 & Baccharis crispa & 14.3 & 0.57 & 1.16 & 0.46 & 0.81 & 67.0 & 88.0 \\
\hline 26 & Myrcia hartwegiana & 14.3 & 0.43 & 1.16 & 0.34 & 0.75 & 57.0 & 67.0 \\
\hline 27 & Mikania lanuginosa & 14.3 & 0.43 & 1.16 & 0.34 & 0.75 & 40.0 & 51.0 \\
\hline 28 & Gaylussacia brasiliensis var. brasiliensis & 14.3 & 0.14 & 1.16 & 0.11 & 0.64 & 34.0 & 34.0 \\
\hline 29 & Hesperozygis rhododon & 14.3 & 0.14 & 1.16 & 0.11 & 0.64 & 81.0 & 81.0 \\
\hline 30 & Senna organensis var. extratropica & 14.3 & 0.14 & 1.16 & 0.11 & 0.64 & 52.0 & 52.0 \\
\hline \multirow[t]{2}{*}{31} & Smilax campestris & 14.3 & 0.14 & 1.16 & 0.11 & 0.64 & 59.0 & 59.0 \\
\hline & Total & 1228.6 & 124.86 & 100 & 100 & 100 & & \\
\hline
\end{tabular}

Table 16. Phytosociological parameters of the grassy physiognomy of the upper montane grasslands in Serra da Pedra Branca do Araraquara, $n=5$. FA: absolute frequency (\%); Co: absolute cover (\%); FR: relative frequency (\%); CoR: relative cover (\%); PI: importance percentual (\%); Hpre: predominant height (cm); Hmax: maximum height $(\mathrm{cm})$.

\begin{tabular}{llrrrrrrc}
\hline & Species & FA & Co & FR & CoR & PI & Hpre & Hmax \\
\hline 1 & Cryptangium triquetrum & 100 & 43.80 & 11.63 & 40.78 & 26.21 & 55.8 & 70.0 \\
2 & Eryngium koehneanum & 100 & 13.80 & 11.63 & 12.85 & 12.24 & 36.6 & 40.6 \\
3 & Tibouchina dubia & 100 & 12.60 & 11.63 & 11.73 & 11.68 & 47.2 & 63.8 \\
4 & Poaceae 12 & 80 & 15.00 & 9.30 & 13.97 & 11.63 & 54.3 & 60.0
\end{tabular}


Table 16 (continuation)

\begin{tabular}{llccccccc}
\hline & Species & FA & Co & FR & CoR & PI & Hpre & Hmax \\
\hline 5 & Mikania lanuginosa & 60 & 2.20 & 6.98 & 2.05 & 4.51 & 49.3 & 58.0 \\
6 & Myrtaceae 3 & 60 & 2.20 & 6.98 & 2.05 & 4.51 & 50.0 & 58.0 \\
7 & Vernonanthura montevidensis & 20 & 6.80 & 2.33 & 6.33 & 4.33 & 74.0 & 112.0 \\
8 & Coccocypselum condalia & 40 & 1.40 & 4.65 & 1.30 & 2.98 & 25.0 & 25.5 \\
9 & Xyris piraquarae & 40 & 1.20 & 4.65 & 1.12 & 2.88 & 56.5 & 59.0 \\
10 & Cyperaceae 4 & 20 & 1.60 & 2.33 & 1.49 & 1.91 & 12.0 & 12.0 \\
11 & Myrcia hartwegiana & 20 & 1.60 & 2.33 & 1.49 & 1.91 & 91.0 & 128.0 \\
12 & Vriesea hoehneana & 20 & 1.60 & 2.33 & 1.49 & 1.91 & 35.0 & 38.0 \\
13 & Cyperaceae 5 & 20 & 0.60 & 2.33 & 0.56 & 1.44 & 50.0 & 52.0 \\
14 & Hesperozygis rhododon & 20 & 0.60 & 2.33 & 0.56 & 1.44 & 65.0 & 70.0 \\
15 & Agarista niederleinii var niederleinii & 20 & 0.40 & 2.33 & 0.37 & 1.35 & 44.0 & 44.0 \\
16 & Baccharis crispa & 20 & 0.40 & 2.33 & 0.37 & 1.35 & 70.0 & 81.0 \\
17 & Senna organensis var. extratropica & 20 & 0.40 & 2.33 & 0.37 & 1.35 & 53.0 & 75.0 \\
18 & Valeriana ulei & 20 & 0.40 & 2.33 & 0.37 & 1.35 & 55.0 & 61.0 \\
19 & Leandra quinquedentata & 20 & 0.20 & 2.33 & 0.19 & 1.26 & 61.0 & 61.0 \\
20 & Miconia lymanii & 20 & 0.20 & 2.33 & 0.19 & 1.26 & 20.0 & 20.0 \\
21 & Pimenta pseudocaryophyllus & 20 & 0.20 & 2.33 & 0.19 & 1.26 & 100.0 & 100.0 \\
22 & Rhynchospora sp. 3 & 20 & 0.20 & 2.33 & 0.19 & 1.26 & 13.0 & 13.0 \\
\hline & Total & 860 & 107.4 & 100 & 100 & 100 & & \\
\hline
\end{tabular}

austrobrasiliensis, in which it showed the fifth highest percentage of importance. Chusquea pinifolia was found in three physiognomies as one of the four most important species.

In Serra da Igreja, Cryptangium triquetrum and Croton mullerianus stood out on both shrubby and grassy physiognomies. We also observed the importance of Tibouchina dubia on both physiognomies. In the grassy physiognomy, Myrsine altomontana was found as the fourth most important species, which was the only time such species was observed among the main species.

The absence of Croton mullerianus among the main species in Serra da Farinha Seca drew our attention, even though it does occur in the area, but it was not found in neither physiognomy of the sampled plots. In the other two sub-ranges (Serra Gigante and Serra P.B. do Araraquara), where C. mullerianus does not stand out in the community, the species was not even detected in the floristic survey (Mocochinski \& Scheer 2008). Due to the low vegetation cover and frequency of C. mullerianus, Mimosa congestifolia species dominates the shrubby physiognomy and also stands out in areas dominated by Chusquea pinifolia. Cryptangium triquetrum, besides being the most important species in the grassy physiognomy also stood out in the shrubby physiognomy. Another important species in this Serra da Farinha Seca is Sticherus sp., which stood out in both shrubby and Chusquea pinifolia physiognomies.

We found three species in the grassy physiognomy of Serra Gigante, however, they were not important in the other subranges studied herewith. Tibouchina hatschbachii and Neomarica caerulea were detected only in Serra Gigante, whereas Paspalum polyphyllum was previously found in only one sampled plot of Serra do Ibitiraquire.

Cryptangium triquetrum and Croton mullerianus were found as the most important species in both grassy and shrubby physiognomies of Serra da Prata, in which Machaerina austrobrasiliensis was also important. Gaylussacia brasiliensis was found for the first time as one of the most important species, ranking in the fourth position of the grassy physiognomy.

Tibouchina dubia and Eryngium koehneanum stood out among the most important species in both grassy and shrubby physiognomies of Serra da Pedra Branca do Araraquara. While Vernonanthura montevidensis was found as one of the most important species only in this mountain range.

Croton mullerianus was the most important species in the shrubby physiognomy within three 
out of the five subranges where such physiognomy was plot-sampled. Cryptangium triquetrum, despite not being the most important species in the shrubby physiognomy, was among the four most important ones within four subranges. Tibouchina dubia was found within three subranges among the most important species in the shrubby physiognomy. Both Mimosa congestifolia and Sticherus sp. were found among the most important species in the shrubby physiognomy within two subranges.

Cryptangium triquetrum was the most important species in the grassy physiognomy within all suveyed

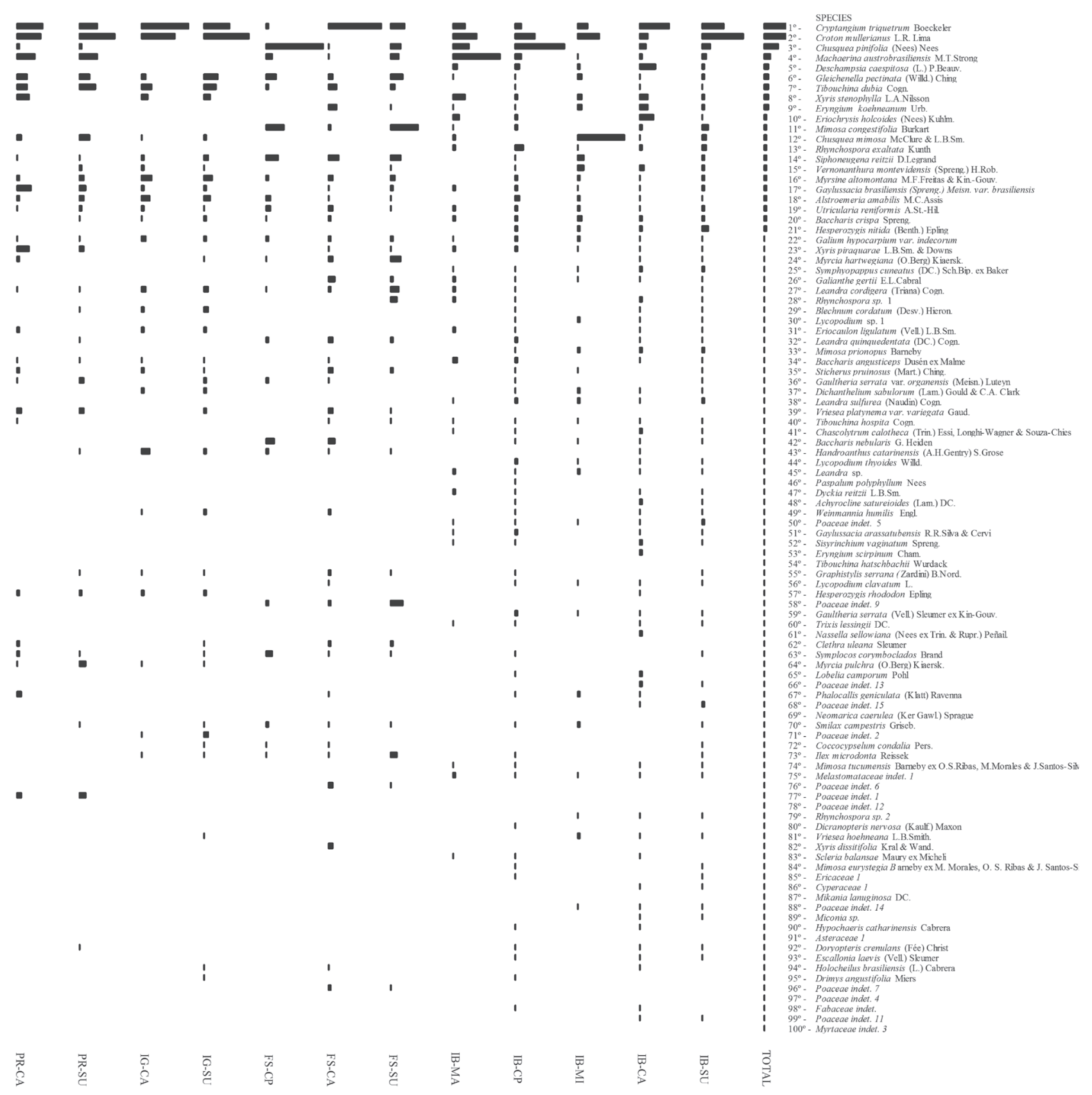

continue

Figure 2. Part 1. The 195 most important species in the phytosociological structure of upper montane grasslands in different physiognomies in mountains of six sub Subranges of Serra do Mar, Southern Brazil; $n=324$ ( $1 \mathrm{~m}^{2}$ plots). Subranges: FS: Farinha Seca; GI: Gigante; PB: Pedra Branca do Araraquara; IB: Ibitiraquire; IG: Igreja; PR: Prata. Physiognomies: CA: Grassy; SU: Shrubby; CP: Chusquea pinifolia; MA: Machaerina austrobrasiliensis; MI: Chusquea mimosa. 
Figure 2 (continuation)

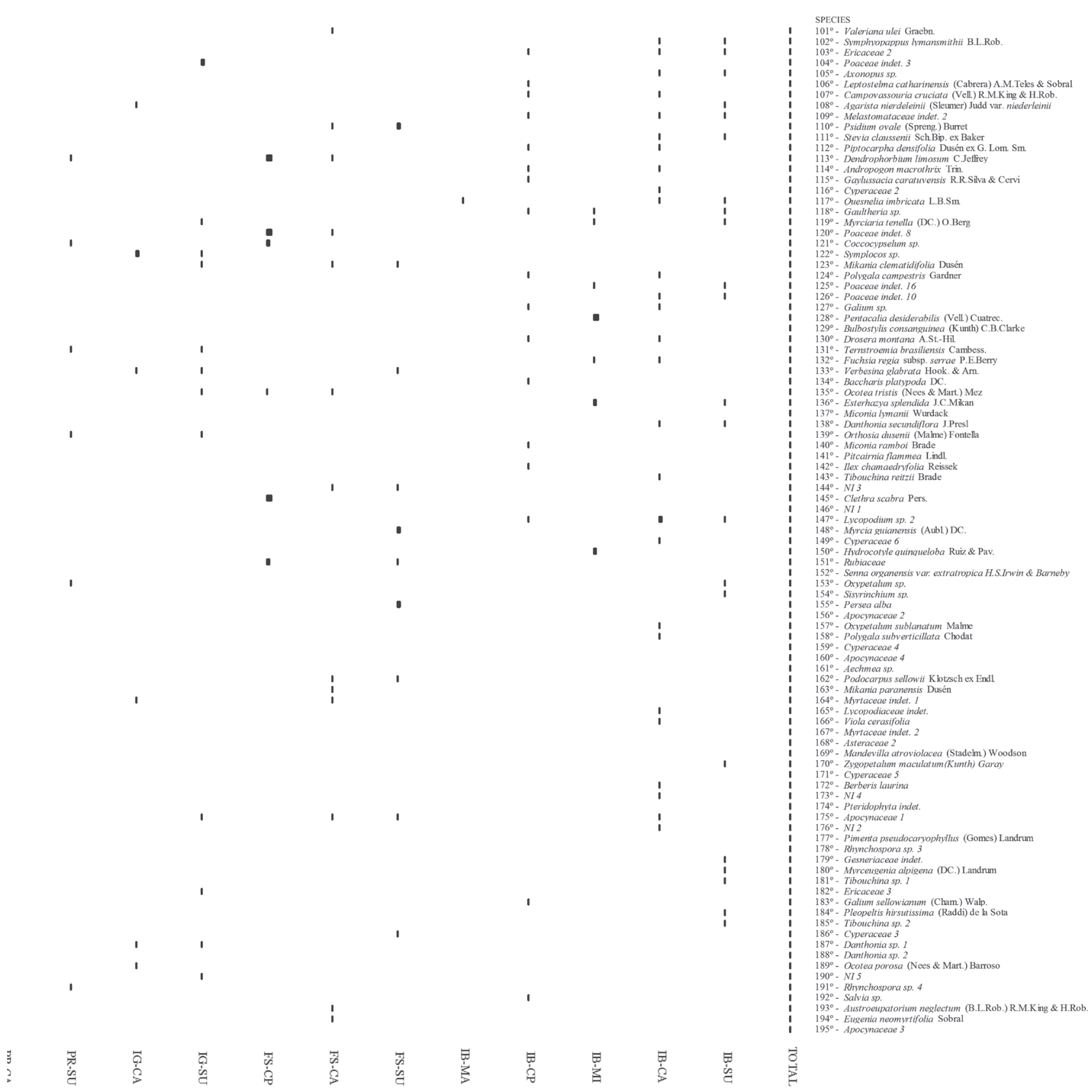

Figure 2. Part 2. The 195 most important species in the phytosociological structure of upper montane grasslands in different physiognomies in mountains of six sub Subranges of Serra do Mar, Southern Brazil; $n=324$ ( $1 \mathrm{~m}^{2}$ plots). Subranges: FS: Farinha Seca; GI: Gigante; PB: Pedra Branca do Araraquara; IB: Ibitiraquire; IG: Igreja; PR: Prata. Physiognomies: CA: Grassy; SU: Shrubby; CP: Chusquea pinifolia; MA: Machaerina austrobrasiliensis; MI: Chusquea mimosa.

subranges. Croton mullerianus, Tibouchina dubia and Eryngium koehneanum also stood out in this physiognomy among the most important species in at least two subranges.

The dominance of one or a few species was a constant in all the subranges as well as their phytophysiognomies. Such pattern has been fairly well-known, in which habitats subjected to extreme environmental conditions such as Restingas (beach ridge vegetation), rupestrian vegetation and upper montane grasslands, tend to be dominated by one or a few species (Scarano 2002, Caiafa \& Silva 2007). 

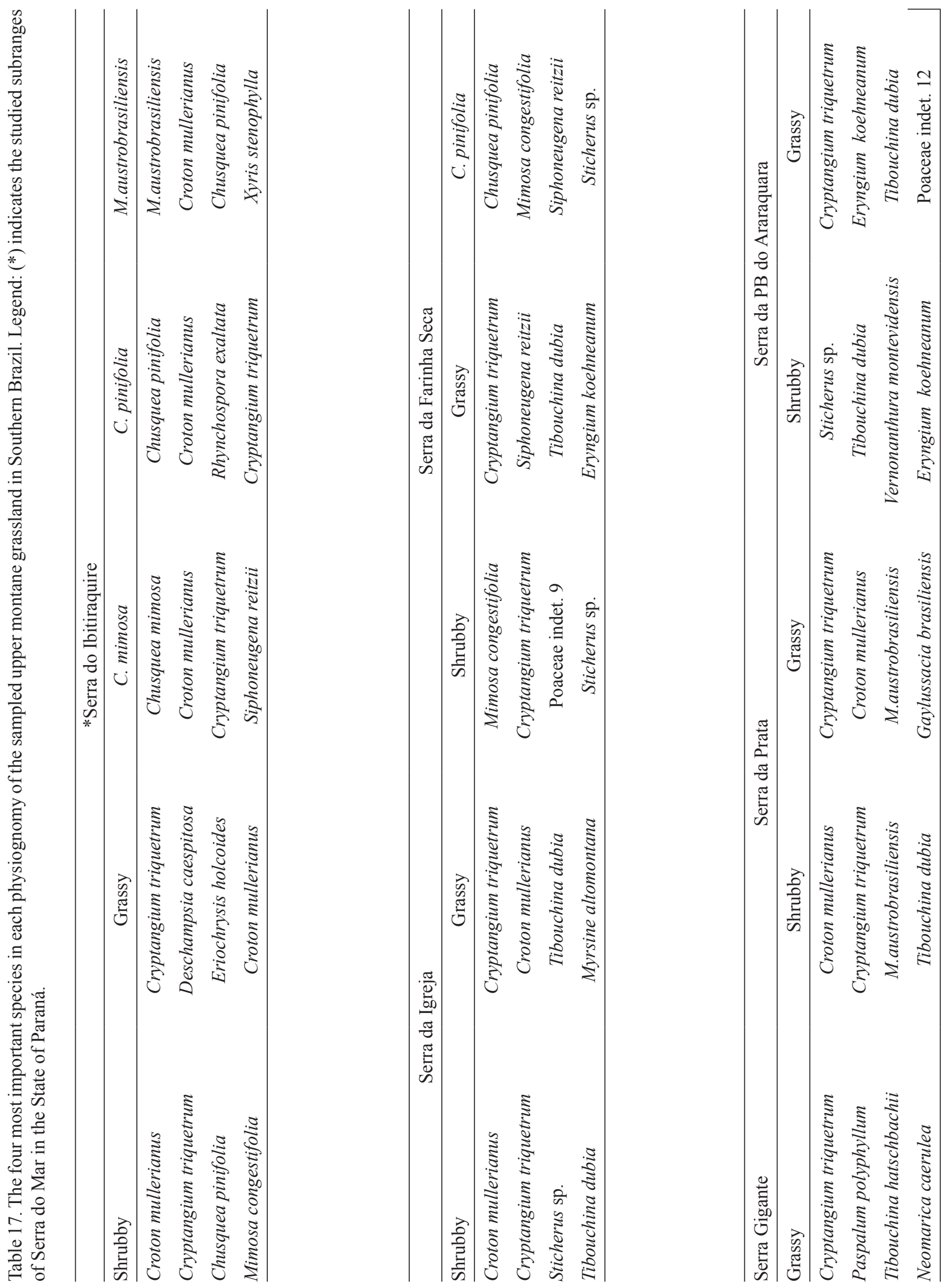
Table 18 summarizes the structural parameters as well as Shannon's Diversity and Evenness indexes ( $\mathrm{H}^{\prime}$ and e) of each physiognomy in the sampled subranges.

The greatest diversity detected in the grassy physiognomy within Serra do Ibitiraquire is mainly due to the high species richness, which is likely caused by increased sampling intensity related to its greater extension and diversity of environments as well as its greater altitudinal gradient. The stretches of shrubby physiognomy within Serra da Farinha Seca and Serra da Pedra Branca do Araraquara showed relatively high values of diversity, despite their relatively lower richness. As a matter of fact, this is caused by the greater evenness in these areas, indicating a more uniform distribution of cover among different species. The grassy physiognomy in Serra da Prata also showed relatively high evenness. Conversely, the Chusquea pinifolia physiognomy in Serra da Farinha Seca showed low evenness values, which evidenced communities where the species of greater cover spreads over most of the relative cover. In this case, Chusquea pinifolia dominated $52 \%$ of the relative cover. In the grassy physiognomy of Serra Gigante, Cryptangium triquetrum also dominated 52\% of the relative cover resulting in a lower community evenness.

For a more detailed analysis of the relationship between species richness and diversity, it would be interesting to standardize the number of plot samples in the physiognomies of each subrange. As the obtention of the diversity index based upon the species relative abundance is considered unviable, given the difficulty of differentiating individuals mainly of grass species ("graminoids"), obtaining the index from the relative cover may prove to be very useful, since this variable of estimates is not only viable, but also acceptable. Furthermore, as individuals from different species coverage may vary greatly (e.g. grasses that propagate by stolons may cover large areas, using a single specimen), the use of the cover is found to be interesting to assess the physiognomic heterogeneity of different communities.

We observed that there is greater similarity between grassy and shrubby physiognomies within Serra da Igreja, and secondarily, the same physiognomies within Serra da Prata (figure 3). These are medium-altitude subranges (1,320-1,450 m asl) among the sampled ones. The physiognomies of the subranges, where the sampled stretches were at lower altitudes (1,000-1,250 m asl), also formed a small group. The three physiognomies of Serra da Farinha Seca were also secondarily linked to this group, and the altitude was slightly higher (1,200-1,400 m asl). All physiognomies of Serra do Ibitiraquire (this subrange is the highest in altitude, stretches between 1,600 and 1,870 $\mathrm{m}$ asl) formed a large group (figure 3). These results indicate a strong relationship between the phytosociological structure of the sampled

Table 18. Parameters for each physiognomy of the sampled upper montane grassland in Southern Brazil. N: number of sampled plots, S: species richness, Co: absolute cover, spp/UA: average number of species per plot, H': Shannon-Wiener index, e: evenness indices.

\begin{tabular}{lccccccc}
\hline Subrange & Physiognomy & $\mathrm{N}$ & $\mathrm{S}$ & Co $(\%)$ & spp/UA & H' (nats/Co) & $\mathrm{e}$ \\
\hline Ibitiraquire & Grassy & 64 & 99 & 109 & 8 & 3.00 & 1.5 \\
Ibitiraquire & Shrubby & 61 & 94 & 125 & 10 & 2.74 & 1.39 \\
Ibitiraquire & Chusquea pinifolia & 56 & 85 & 125 & 10 & 2.60 & 1.35 \\
Farinha Seca & Grassy & 22 & 53 & 148 & 12 & 2.39 & 1.39 \\
Igreja & Shrubby & 27 & 50 & 149 & 10 & 2.30 & 1.34 \\
Ibitiraquire & Chusquea mimosa & 9 & 46 & 136 & 10 & 2.34 & 1.41 \\
Farinha Seca & Shrubby & 10 & 42 & 135 & 12 & 2.77 & 1.71 \\
Ibitiraquire & Machaerina austrobrasiliensis & 10 & 36 & 102 & 9 & 2.18 & 1.40 \\
Prata & Shrubby & 15 & 36 & 121 & 11 & 2.48 & 1.59 \\
Igreja & Grassy & 14 & 33 & 151 & 10 & 2.03 & 1.80 \\
PBA & Shrubby & 7 & 31 & 125 & 12 & 2.69 & 1.80 \\
Prata & Grassy & 7 & 30 & 125 & 11 & 2.54 & 1.72 \\
Farinha Seca & Chusquea pinifolia & 5 & 29 & 134 & 11 & 0.73 & 0.50 \\
Gigante & Grassy & 12 & 23 & 112 & 8 & 1.81 & 1.33 \\
PBA & Grassy & 5 & 22 & 107 & 9 & 1.97 & 1.47 \\
\hline
\end{tabular}


communities and the colonization opportunities for different populations based on the geographic location of each subrange, as well as their potential to act as a dispersal barrier (selective) for some species. In addition to the limitations imposed by geographical isolation, as the ones occurring on "inselbergs" (Porembski 2007), the altitude conditioning factors (e.g. temperature gradients, geomorphology/soils, formation of histic soil horizons, etc.), may also exert considerable influence on some taxa.

As we mentioned earlier, there is still scarce information about the structure and floristic composition as well as the diversity of upper montane ecosystems. Therefore, further studies and surveys are required in order to obtain a more widely applicable ecological knowledge. However, we conducted a brief analysis relating to upper montane grasslands of the present study with other vegetation studies about altitude rocky outcrops in Serra da Mantiqueira (Southeastern Brazil) (Caiafa \& Silva 2007, Ribeiro et al. 2007, Tinti et al. 2015) as well as two sites of Serra do Mar (Araras \& Madalena) in the State of Rio de Janeiro (Meirelles et al. 1999). We learned that neither of the species found in the upper montane grasslands within Serra do Mar in Paraná was found in these two areas in Rio de Janeiro. Perhaps because such species might be more adapted to grasslands than rocky outcrops. Based on studies carried out at Serra da Mantiqueira only two species were found (Caiafa \& Silva 2007) to approximately two dozen species (Tinti et al. 2015) in common with the ones found within the subranges studied in Paraná, which in

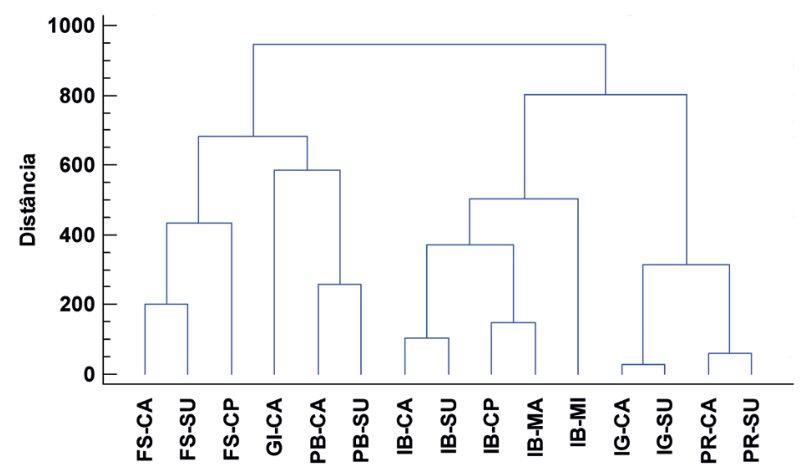

Figure 3. Clusters for upper montane grassland physiognomies in Southern Brazil using phytosociological importance values. Ward's method, City Block distances. Subranges: FS: Farinha Seca; GI: Gigante; PB: Pedra Branca do Araraquara; IB: Ibitiraquire; IG: Igreja; PR: Prata. Physiognomies: CA: Grassy; SU: Shrubby; CP: Chusquea pinifolia; MA: Machaerina austrobrasiliensis; MI: Chusquea mimosa. addition to the distances between them, may be related to the surrounding phytogeographic aspects. The floristic study reported by Tinti et al. (2015) (Serra do Brigadeiro - Mantiqueira, in the State of Minas Gerais) is in alignment with the grasslands of our study, for instance, the following taxa: Achyrocline satureoides, Stevia clausseni, Gaultheria serrata, "Croton mullerianus"(Croton splendidus), Hesperozygis nitida, Lycopodium clavatum, Doryopteris crenulans and Galium hypocarpium. Whereas, the phytosociological analysis by Caiafa \& Silva (2007), shares only Esterhazya splendida, ranking in the twenty-fourth position in terms of structural importance value, in Serra da Mantiqueira (Minas Gerais) as well as the physiognomy dominated by Chusquea mimosa within Serra do Ibitiraquire (Serra do Mar) in Paraná.

Although the phytogeographical study by Safford (2007) has given greater emphasis to genus level, by using floristic lists for upper montane grasslands (high altitude grasslands) of subranges such as Itatiaia, Orgãos and Caparaó (Southeastern Brazil), showed considerable sharing in this taxonomic level, for example: Cryptangium, Croton, Chusquea, Tibouchina, Hesperozygis, Alstroemeria, Esterhazya, Xyris, Mimosa, Gaultheria and Utricularia. Based on Jaccard's index of similarity, Safford detected the following between upper montane grasslands (Southeastern areas) and Aparados da Serra Geral (Southern areas): $41.4 \%$ for genera and $9.1 \%$ for species.

Although a considerable number of common species were found in the upper montane grasslands within Serra do Mar in Paraná, the survey conducted by Falkenberg (2003) in upper montane environments within Aparados da Serra Geral in both States of Santa Catarina and Rio Grande do Sul, the author studied rupicolous vegetations adjacent to jetties and extremely steep slopes of basalt, which are quite different environments, and therefore, causing a major limiting factor for comparisons.

Currently, there have been several botanical surveys made available in the literature. Nevertheless, their updating, site specification and structural physiognomy of the habitat are needed so that other research studies may provide comparisons. Actually, there is a gap on the phytosociological structure of upper montane grasslands in Brazil. Such knowledge plays a very important role to better understand these environments.

\section{Conclusions}

The upper montane grasslands within Serra do Mar in Paraná occur in higher places of the mountains, 
but they can also be found at lower altitudes, unlike what has been reported in other studies (over $1,200 \mathrm{~m}$ asl). Serra Gigante, for instance, where upper montane grasslands dominate mountain ridges between 1,000 and 1,070 $\mathrm{m}$ asl, even though they present some different species.

Cryptangium triquetrum and Croton mullerianus comprise the main species sampled in the upper montane grasslands of Serra do Mar in Paraná. The first species was the most important one in all the grassy stretches (subranges), while the second species stood out in the shrubby physiognomy within three sampled subranges. Chusquea pinifolia, Machaerina austrobrasiliensis, Deschampsia caespitosa, Gleichenella pectitata, Tibouchina dubia, Xyris stenophylla, Eryngium koehnearum and Eriochrysis holcoides also stood out in several stretches and physiognomies.

Phytosociological studies about grassland vegetation are quite scarce in Brazil. This situation still difficults better definitions of these environments on a continental scale, although our findings can serve as an important source of data for further ecological studies. Despite incipient, a certain similarity to genus, the comparisons among other upper montane vegetations mainly on rocky outcrops (considering the limitations of the analyzed sites) indicate that, there are likely great floristic-structural differences between the upper montane grasslands of Southern and Southeastern Brazil. In order to explain the occurrence of different physiognomies found in our study, as well as, in other surveys on upper montane ecosystems, further investigations are required, particularly regarding soils and geomorphology.

Finally, most of the species found in this study are not cited in the List of Species (the so called "indicator species") relating to Altitude Grasslands (campos de altitude montanos e altomontanos) of Southern Brazil (CONAMA Resolution 423/2010). This fact indicates that, the data about the structure of well-preserved native communities (such as the ones studied herewith) can help understanding the ecology of ecosystems as well as strategies for their protection and management.

\section{Acknowledgments}

This study was supported and sponsored by Fundação Grupo O Boticário de Proteção à Natureza FGBPN (Projeto Altomontana - A Floresta Ombrófila Densa Altomontana e os Refúgios Vegetacionais Altomontanos no Paraná). The Authors wish to thank
Sociedade Fritz Müller de Ciências Naturais for supporting the project as well.

We also would like to thank for their support and help: Ruddy Proença, Samuel Arruda, Sandro M. Silva, Gustavo Martinelli, Carlos Vellozo Roderjan, Gustavo Gatti, Gilberto Tiepolo, Osmar S. Ribas, Gert G. Hatschbach, Juarez Cordeiro, Edimilson Costa, Eraldo Barboza, Eduardo Lozano, Renato Goldenberg, Paulo Labiak, Armando C. Cervi, Élide dos Santos, Marília Borgo, Miriam Kaehler, Marcelo Brotto, Carina Kozera, Rodrigo A. Kersten, and O. Vieira for translating most of the manuscript, as well as the anonymous reviewers of the journal.

\section{Literature cited}

Alves, R.J.V., Silva, N.G., Oliveira, J.A. \& Medeiros, D. 2004. Circumscribing campo rupestre - megadiverse Brazilian rocky montane savanas. Brazilian Journal of Biology 4: 355-362.

Aximoff, I. 2010. O que Perdemos com a Passagem do Fogo pelos Campos de Altitude do Estado do Rio de Janeiro? Biodiversidade Brasileira 1: 180-200.

Behling, H. 1997a. Late Quaternary vegetation, climate and fire history of the Araucaria forest and campos region from Serra Campos Gerais, Paraná (South Brazil). Review of Palaeobotany \& Palynology 97: 109-121.

Behling, H. 1997b. Late Quaternary vegetation, climate and fire history from the tropical mountain region of Morro de Itapeva, SE Brazil. Palaeogeography, Palaeoclimatology, Palaeoecology 129: 407-422.

Behling, H., Jeske-Pieruschka, V., Schüler, L. \& Pillar, V.P. 2009. Dinâmica dos campos no sul do Brasil durante o Quaternário tardio. In: S.C.Valerio Pillar, Z.M. Müller, A.V.A. de Souza (eds.). Campos Sulinos - conservação e uso sustentável da biodiversidade. Ministério do Meio Ambiente, Jaques, Brasília, pp.12-25.

Bigarella, J.J. 1978. A Serra do Mar e a porção oriental do estado do Paraná, um problema de segurança ambiental e nacional (contribuição à geografia, geologia e ecologia regional). SEP, ADEA, Curitiba.

Braun-Blanquet, J. 1979. Fitosociologia. H. Blume, Madrid.

Caiafa, A.N. \& Silva, A.F. 2005 Composição florística de um campo altomontano no Parque Estadual da Serra do Brigadeiro, Minas Gerais - Brasil. Rodriguésia 56: $163-173$.

Caiafa, A.N. \& Silva, A.F. 2007. Structural analysis of the vegetation on a highland granitic rock outcrop in Southeast Brazil. Revista Brasileira de Botânica 30: 657-664.

Carvalho, D.A. 1992. Flora fanerogâmica de campos rupestres da Serra da Bocaina, Minas Gerais: caracterização e lista de espécies. Ciência e Prática, 16: 97-122. 
Falkenberg, D.B. 2003. Matinhas nebulares e vegetação rupícola dos Aparados da Serra Geral (SC/RS), sul do Brasil. Tese de Doutorado, Universidade de Campinas, Campinas.

Flenley, J.R. 1995. Cloud Forest, the Massenerhebung effect, and ultraviolet insolation. In: L.S. Hamilton, J.O. Juvik \& F.N. Scatena (eds.). Tropical montane cloud forests. Springer Verlag, New York, pp. 150-155.

Flora do Brasil 2020 (em construção). 2016. Jardim Botânico do Rio de Janeiro. Available in http:// floradobrasil.jbrj.gov.br/ (access in 19-IV-2016).

Ferri, M.G. 1980. Vegetação Brasileira. Editora da Universidade de São Paulo, São Paulo.

Forzza, R.C. et al. (orgs.). 2010. Catálogo de plantas e fungos do Brasil. 2 vols. Andrea Jakobsson Estúdio, Jardim Botânico do Rio de Janeiro, Rio de Janeiro.

Garcia, R.J.F. \& Pirani, J.R. 2005. Análise florística, ecológica e fitogeográfica do Núcleo Curucutu, Parque Estadual da Serra do Mar (São Paulo, SP), com ênfase nos campos junto à crista da Serra do Mar. Hoehnea 32: 1-48.

Grubb, P.J. 1971. Interpretation of the "Massenerhebung" effect on tropical mountains. Nature 229: 44-45.

Han, F., Yao, Y., Dai, S., Wang, C., Sun, R., Xu, J., \& Zhang, B. 2012. Mass elevation effect and its forcing on timberline altitude. Journal of Geographical Sciences 22: 609-616.

IBGE - Instituto Brasileiro de Geografia e Estatística. 1992. Manual técnico da vegetação brasileira (Manuais Técnicos de Geociências 1). IBGE, Rio de Janeiro.

IBGE - Instituto Brasileiro de Geografia e Estatística. 2012. Manual Técnico da Vegetação Brasileira (Manuais Técnicos em Geociências 1). 2 ed. revista e ampliada. IBGE, Rio de Janeiro.

Maack, R. 1968. Geografia física do Estado do Paraná. Universidade Federal do Paraná, Curitiba.

Martinelli, G. 1996. Campos altomontanos. Index, Rio de Janeiro.

Meireles, L.D., Kinoshita, L.S. \& Shepherd, G.J. 2014. Composição florística da vegetação altimontana do distrito de Monte Verde (Camanducaia, MG), Serra da Mantiqueira Meridional, Sudeste do Brasil. Rodriguésia 65: 831-859.

Meirelles, S.T., Pivello, V.R. \& Joly, C.A. 1999. The vegetation of granite rock outcrops in Rio de Janeiro, Brazil, and the need for its protection. Environmental Conservation 1:10-20.

Ministério do Meio Ambiente. 1993. Conselho Nacional do Meio Ambiente. 1993. Resolução CONAMA 10, de 01-X-1993. Estabelece os parâmetros básicos para análise dos estágios de sucessão de Mata Atlântica. Diário Oficial da União, no 209, 02-XI-1993, pp. 16497-16498.
Ministério do Meio Ambiente. 2010. Conselho Nacional do Meio Ambiente. 2010. Resolução CONAMA 423, de 12-IV-2010. Dispõe sobre parâmetros básicos para identificação e análise da vegetação primária e dos estágios sucessionais da vegetação secundária nos Campos de Altitude associados ou abrangidos pela Mata Atlântica. Diário Oficial da União, nº 69, 13-IV-2010, pp. 55-57.

Mocochinski, A.Y. \& Scheer, M.B. 2008. Campos de altitude na Serra do Mar paranaense: aspectos florísticos. Floresta 38: 625-640.

Oliveira-Filho, A.T. \& Fluminhan-Filho M. 1999. Ecologia da vegetação do Parque Florestal Quedas do Rio Bonito. Cerne 5: 51-64.

Overbeck G.E., Müller S.C., Fidelis A., Pfadenhauer J., Pillar V.D., Blanco C.C., Boldrini I.I., Both R. \& Forneck E.D. 2007. Brazil's neglected biome: The South Brazilian Campos. Perspectives in Plant Ecology, Evolution and Systematics 9: 101-116.

Péllico Neto S., Brena A.B. 1997. Inventário florestal. Editorado pelos autores, Curitiba.

Porembski, S. 2007. Tropical inselbergs: habitat types, adaptive strategies and diversity patterns. Revista Brasileira de Botânica 30: 579-586.

Presidência da República. 2006. Lei Federal 12.428, de 26-XII-2006. Dispõe sobre a utilização e proteção da vegetação nativa do Bioma Mata Atlântica, e dá outras providências. Diário Oficial da União, 26-XII-2006.

PRÓ-ATLÂNTICA - Programa de Proteção da Floresta Atlântica. 2002. Projeto Carta Geológica. Cartas: MI-2844-2 Ariri, MI-2843-1 Represa do Capivari, MI2843-3 Morretes, MI-2858-1 Mundo Novo e MI-2858-3 Pedra Branca do Araraquara. Escala 1:50000. Curitiba.

Ribeiro, K.T., Medina, B.M.O. \& Scarano, F.B. 2007. Species composition and biogeographic relations of the rock outcrop flora on the high plateau of Itatiaia, SE-Brazil. Revista Brasileira de Botânica 30: 623-639.

Rizzini, C.T. 1979. Tratado de fitogeografia do Brasil. HUCITEC, São Paulo.

Roderjan, C.V. 1999. Caracterização da vegetação dos Refúgios Vegetacionais Altomontanos (campos altomontanos) nas serras dos órgãos e do Capivari no Estado do Paraná. Relatório Técnico do CNPq, Curitiba.

Roderjan, C.V. 1994. A Floresta Ombrófila Densa Altomontana no morro Anhangava, Quatro Barras, PR Aspectos Climáticos, Pedológicos e Fitossociológicos. Tese de Doutorado, Universidade Federal do Paraná, Curitiba.

Roderjan, C.V., Galvão, F., Kuniyoshi, Y.S. \& Hatschbach, G.G. 2002. As unidades fitogeográficas do estado do Paraná. Ciência \& Ambiente 24: 75-92.

Safford, H.D. 1999a. Brazilian Paramos I. An introduction to the physical environment end vegetation of the campos altomontanos. Journal of Biogeography 26: 693-712. 
Safford, H.D. 1999b. Brazilian Páramos II. Macro and mesoclimate of the campos altomontanos and affinities with high mountain climates of tropical Andes and Costa Rica. Journal of Biogeography 26: 713-737.

Safford, H.D. 2001. Brazilian Paramos. III. Patterns and Rates of Postfire Regeneration in the Campos de Altitude. Biotropica 33: 282-302.

Safford, H.D. 2007. Brazilian Paramos. IV. Phytogeography of the campos de altitude. Journal of Biogeography 34: 1701-1722.

Scarano, F.R. 2002. Structure, Function and Floristic Relationships of Plant Communities in Stressful Habitats Marginal to the Brazilian Atlantic Rainforest. Annals of Botany 90: 517-524.

Scheer, M.B. \& Mocochinski, A.Y. 2009. Floristic composition of four tropical upper montane rain forests in Southern Brazil (in portuguese). Biota Neotropica 9: 51-70.

Scheer, M.B., Curcio, G. \& Roderjan, C.V. 2011. Environmental functionalities of upper montane soils in Serra da Igreja, Southern Brazil (in portuguese). Revista Brasileira de Ciência do Solo 35: 1013-1026.

Scheer, M.B., Mocochinski, A.Y. \& Roderjan, C.V. 2011. Tree component structure of tropical upper montane rain forests in Southern Brazil (in portuguese). Acta Botanica Brasilica 25: 735-750.

Scheer M.B., Curcio, G. \& Roderjan, C.V. 2013. Carbon and Water in Upper Montane Soils and Their Influences on Vegetation in Southern Brazil. Hindawi Publishing Corporation, pp. 1-12.
Scheer, M.B., Curcio, G.R. \& Roderjan, C.V. 2013. The Late Holocene upper montane cloud forest and high altitude grassland mosaic in the Serra da Igreja, Southern Brazil. Anais da Academia Brasileira de Ciências 85: 769-783.

Scheer, M.B., Pereira, N.V., Behling, H., Curcio, G.R. \& Roderjan, C.V. 2014. Nine thousand years of upper montane soil/vegetation dynamics from the summit of Caratuva Peak, Southern Brazil. Journal of South American Earth Sciences 56: 365-375.

Tinti, B.V., Schaefer, C.E.R.G., Nunes, J.A., Rodrigues, A.C., Fialho, I.F. \& Neri, A.V. 2015. Plant diversity on granite/gneiss rock outcrop at Pedra do Pato, Serra do Brigadeiro State Park, Brazil. Check List 11: 1-8.

Tramujas, A.P. 2000 A vegetação dos campos altomontanos na região do Ibitiraquire - Serra do Mar - Municípios de Antonina, Morretes e Campina Grande do Sul, Paraná. Dissertação de Mestrado, Universidade Federal do Paraná, Curitiba.

Vashchenko, Y., Piovesan, R.P, Lima, M.R \& Favaretto, N. 2007. Solos e Vegetação dos Picos Camacuã, Camapuã e Tucum - Campina Grande Do Sul-PR. Scientia Agraria 8: 411-419.

Vasconcelos, M.F.D. 2011. O que são campos rupestres e campos de altitude nos topos de montanha do Leste do Brasil? Brazilian Journal of Botany 34: 241-246.

Veloso, H.P., Rangel Filho, A.L.R. \& Lima, J.C.A. 1991. Classificação da vegetação brasileira, adaptada a um sistema universal. IBGE, Rio de Janeiro.

Wisniewski, C., Tempesta, P.B., Rodrigues, V.M.R. 2005. Solos e vegetação em uma topossequência do morro Mãe Catira, alto da Serra da Graciosa, Morretes PR.. In: Anais XXX Congresso Brasileiro de Ciência do Solo, Minas Gerais. 